\title{
Control of synaptic plasticity in deep cortical networks
}

4

Pieter R. Roelfsema ${ }^{1,2,3}$ and Anthony Holtmaat ${ }^{4}$

${ }^{1}$ Department of Vision and Cognition, Netherlands Institute for Neuroscience, Royal Netherlands Academy of Arts and Sciences

${ }^{2}$ Department of Integrative Neurophysiology, Centre for Neurogenomics and Cognitive Research, VU University

${ }^{3}$ Psychiatry Department, Academic Medical Center, Amsterdam, The Netherlands

${ }^{4}$ Department of Basic Neurosciences, Geneva Neuroscience Center, Faculty of Medicine, University of Geneva, Geneva, Switzerland.

\section{Correspondence to P.R.R.}

p.roelfsema@nin.knaw.nl

\section{Abstract}

Humans and many other animals have an enormous capacity to learn about sensory stimuli and to master new skills. Yet, many of the mechanisms that enable us to learn remain to be understood. One of greatest challenges of systems neuroscience is to explain how synaptic connections change to support maximally adaptive behaviour. Here, we give an overview of factors that determine the change in the strength of synapses, with a focus on synaptic plasticity in sensory cortices. We review the influence of neuromodulators and feedback connections in synaptic plasticity, and suggest a specific framework in which these factors can interact to improve the functioning of the entire network.

\section{Introduction}

How does a neuron in the sensory or association cortex optimize the strength of its synapses to improve the performance of the entire brain network? In computational neuroscience, the task of determining the connections that matter for behaviour is known as the 'credit assignment problem'1,2. For artificial neural networks, powerful 
methods exist to solve this problem ${ }^{3,4}$. However, how it is solved in the brain is an important but still open question.

Suppose that an animal recognizes a particular stimulus, selects a response and is then rewarded unexpectedly. Synapses in association and motor cortices should change, to promote the selection of the same action if the same stimulus reappears in the future. Furthermore, learning should sharpen representations of the stimulus in sensory cortices if slightly different stimuli require distinct responses.

In this Review, we discuss biologically plausible learning rules that may enable synapses to change in a manner that optimizes behavioural outcome. We focus on synaptic plasticity in sensory cortices, and review frameworks in which learning relies on modifiers of synaptic plasticity. The first modifying factor is a feedback signal from the response-selection processing stage back to association and sensory cortices that informs neurons about the action that was selected. This feedback signal leads to the 'tagging' of synapses and gates their plasticity. The second modifying factor is the release of neuromodulators, which, among other functions, inform synapses about reward prediction errors; that is, whether the outcome of an action was better or worse than expected. We discuss how the combination of feedback connections and neuromodulators permit new learning rules that promote future actions that lead to more reward, and enable 'deep learning' in the brain.

\section{Changing the strength of synapses}

In 1949, Donald O. Hebb ${ }^{5}$ proposed that the change in the strength of a synapse depends on presynaptic and postsynaptic activity. He phrased this hypothesis as follows: "when an axon of cell $A$ is near enough to excite a cell $B$ and repeatedly or persistently takes part in firing it, some growth process or metabolic change takes place in one or both cells such that $A$ 's efficiency, as one of the cells firing $B$, is increased". Hebb's rule can be formalized as follows:

$$
\Delta w_{i, j}=\beta \cdot f_{i}\left(a_{i}\right) \cdot f_{j}\left(a_{j}\right)
$$

$\Delta w_{i, j}$ is the change in the strength of the connection between neurons $i$ and $j, \beta$ is the learning rate parameter and determines the magnitude of the change, and $f_{i}\left(a_{i}\right)$ and $f_{j}\left(a_{j}\right)$ are functions that depend on presynaptic activity $\left(a_{i}\right)$ and postsynaptic activity $\left(a_{j}\right)$.

A wealth of evidence supports Hebb's rule ${ }^{6}$, but researchers realize that the rule is incomplete if the aim is to select appropriate actions, because the rule is ignorant about the usefulness of the network's output. In animals, rewards and punishments influence learning such that behaviours that lead to reward are reinforced and behaviours that result in aversive outcomes are inhibited. 
The influence of theories of reinforcement learning [G] $]^{1}$ increased tremendously when it became clear that neuromodulatory systems, such as the dopaminergic system ${ }^{7}$, code for unexpected reward. In reinforcement learning theory, unexpected rewards and punishments give rise to reward-prediction errors [G] (RPEs) ${ }^{1,8}$. The RPE is positive if the animal receives more reward than expected and negative if the outcome is disappointing. Reinforcement learning theories have proposed that the coincident activity of presynaptic and postsynaptic neurons induces eligibility traces [G] at synapses that determine whether the synapse will undergo plasticity in case of an RPE. Eligibility traces correspond to synaptic tags [G], which are biochemical markers at synapses that are induced by the coincident pre- and postsynaptic activity but can be maintained for some time after the neurons stopped firing 1,9-13. Studies have started to elucidate the molecular identity of these synaptic tags ${ }^{14,15}$ but many discoveries remain to be made.

A positive RPE, for example signalled by the dopamine released from the substantia nigra and ventral tegmental area, is a well-suited signal to strengthen these tagged synapses, because it increases the probability that rewarded actions will be taken again in the future. By contrast, a negative RPE should decrease the strength of tagged synapses. Neuromodulatory systems, including the dopaminergic system, project rather diffusively to the cortex and subcortical structures, suggesting that their signals are conferred globally. The introduction of the RPE as a factor to the Hebbian rule results in the following plasticity rule ${ }^{11,16-19}$ :

$$
\Delta w_{i, j}=\beta \cdot f_{i}\left(a_{i}\right) \cdot f_{j}\left(a_{j}\right) \cdot R P E
$$

Here, we refer to the influence of neuromodulatory signals as 'plasticity-steering' effects.

Another factor that determines learning is selective attention. This is intuitive - we learn more if we pay attention ${ }^{20-22}$. A formal way to test the role of attention in learning uses the redundant-relevant cue paradigm ${ }^{20,21,23}$, in which subjects learn through trial-and-error to map stimuli onto responses. In each trial, participants see multiple stimuli that are all informative about the desired response, such that much of the information is redundant, but the participants pay attention to only one of the stimuli, and learn only about the attended stimuli and not the unattended ones. This is remarkable, because unattended stimuli are paired with the same behavioural responses and are associated with the same RPEs as the attended stimuli. Only under special conditions can perceptual learning occur without attention ${ }^{24}-$ for example, if stimuli are very weak. These weak stimuli appear to escape from the attentional control mechanisms that would otherwise suppress the plasticity of nonattended items ${ }^{25}$.

$$
\text { The attentional signals that gate learning could originate from brain areas in the }
$$
motor and frontal cortex that select behavioural responses. Action selection is 
113 invariably associated with an attention shift ${ }^{26}$ that, through feedback connections,

114 reaches the neurons in sensory cortices that code for the features that caused the

115 action ${ }^{27}$. Introducing attention signals into the learning rule gives

$116 \quad \Delta w_{i, j}=\beta \cdot f_{i}\left(a_{i}\right) \cdot f_{j}\left(a_{j}\right) \cdot R P E \cdot F B_{j}$

117 where $F B_{j}$ is the feedback from higher brain regions that gate the plasticity of

118 synapses onto neuron $j$. We will refer to the effect of $F B_{j}$ as 'gating' because it varies

119 between zero (not attended) and one (fully attended) and is always positive (unlike

120 the 'steering' RPE signal, which can change sign).

121 Figure 1 illustrates the main ideas underlying this learning rule ${ }^{25,28}$. Stimulus

122 information first propagates from sensory cortex to motor cortex during a

123 feedforward processing phase (Fig. 1) ${ }^{29}$. The motor cortex selects an action and uses

124 feedback connections to highlight representations in lower-level cortices that

125 provided input for the action ${ }^{30}$. The feedback connections induce synaptic tags (also

126 known as eligibility traces) that gate plasticity. The placement of tags and their

127 strength depends on presynaptic and postsynaptic activity $f_{i}\left(a_{i}\right)$ and $f_{j}\left(a_{j}\right)$ and on

128 the feedback $F B_{j}$. In this framework, different actions would activate different

129 feedback connections and cause distinct patterns of synaptic tags, ensuring that the

130 credit (or blame) is assigned to those synapses that mattered for the stimulus-

131 response mapping. The tags should persist until the RPE signal becomes available.

132 Neuromodulators signalling the computed RPE interact selectively with tagged

133 synapses to modify their strength.

134 Learning rule (3) permits the training of networks with many layers between the

135 sensory and motor cortices. If the strength of the feedback connections is

136 proportional to that of the feedforward connections, a property that can emerge

137 during learning ${ }^{28,31}$, is equivalent to the so-called error-backpropagation rule [G] ${ }^{32}$

138 that is used to train networks with many layers ${ }^{3}$. Such deep artificial neural networks

139 have achieved excellent and sometimes even superhuman performance in image

140 recognition tasks ${ }^{4}$ and computer games ${ }^{33}$. Thus, although the error-backpropagation

141 rule was previously thought to be biologically unrealistic ${ }^{34}$, new insights suggest that

142 learning rule (3) can be implemented by the brain to enable forms of deep learning

143 (Box 1).

144 Below, we review the corticocortical and corticosubcortical connections that may

145 enable learning rule (3). We then discuss how learning changes the representation of

146 stimuli in sensory and association cortices and review mechanisms for controlling

147 plasticity.

148

149 Sensory and association cortex 
150 The cortex contains a vast network of circuits for local and long-range interactions

151 (Fig. 2a,b). Cortical areas are composed of columns, and the neuronal subtypes and

152 local connectivity patterns in different areas are similar ${ }^{35,36}$. Cortical areas can be

153 arrange in a hierarchical manner, in which lower-order cortical regions (Level I in Fig.

154 2b) feed information forward to higher-order areas (Level II in Fig. 2b), and higher-

155 order regions can feed information back to lower-order cortex ${ }^{37}$. When going up in

156 the hierarchy, the neuronal receptive-field properties become more complex ${ }^{37,38}$.

157 The principles of cortical organization and connectivity have been excellently

158 reviewed elsewhere ${ }^{39-46}$. Here, we summarize key aspects of cortical organization

159 that relate to the feedforward and feedback streams and are relevant to

160 understanding plasticity rules in hierarchical networks.

\section{Feedforward and feedback connections}

163 There are laminar differences as to where feedforward and feedback inputs originate 164 and terminate 37,43 (Fig. 2). Anatomical and neurophysiological studies have revealed 165 that sensory inputs relayed by the thalamus initially activate neurons in L4 and L6 of 166 sensory cortices in the primate $e^{47-50}$, with inputs in $L 3$ and $L 5$ in rodents as well ${ }^{51,52}$.

167 This input then rapidly propagates to the other layers so that neurons in all layers

168 are activated by the sensory input. There is a feedback system within the cortical

169 column, where strong feedback originates from L6 and predominantly suppresses

170 activity ${ }^{53,54}$ by activating inhibitory neurons ${ }^{55}$.

171 Sensory areas also receive feedback connections from higher cortical areas, which

172 mostly provide input to superficial layers (L1-3) and parts of L5 (Fig 2). Hence,

173 whereas interareal feedforward inputs target $L 4$, interareal feedback inputs target

174 the apical tufts of $L 2 / 3$ and $L 5$ pyramidal cells ${ }^{56,57}$, as well as inhibitory ${ }^{58}$ and

175 disinhibitory microcircuits ${ }^{59,60}$. These features may have important consequences for

176 the role of feedback connections in synaptic plasticity (discussed later).

177 Cortical areas also interact with one another indirectly via the thalamus. Cortical

178 neurons in L5 that project to the brainstem send collaterals to higher-order thalamic

179 matrix nuclei (as opposed to the first-order, sensory-specific core nuclei), which, in

180 turn, provide feedforward input to $L 4$ in higher-order cortical areas ${ }^{39,61-63}$.

181 Furthermore, projections from higher-order thalamic nuclei also feed information

182 back to lower-order cortical areas (Fig. 2) ) $^{57,64}$, where they target L1 and L5 ${ }^{61,65-67}$.

183 These feedforward and feedback routes through the thalamus permit the integration

184 of sensory information from the periphery ${ }^{68-71}$ with information from the association

185 and motor cortices ${ }^{39,64,72,73}$.

186 Pharmacological studies have demonstrated that feedforward inputs drive

187 postsynaptic activity by activating AMPA receptors (AMPARs). By contrast, the

188 synapses of many feedback connections modulate firing rates mainly via NMDA 
receptors (NMDARs) ${ }^{74,75}$ and metabotropic glutamate receptors ${ }^{39,76}$. Consistent with this, microstimulation of higher-order thalamic nuclei in mice induces robust NMDAR mediated responses in cortical pyramidal neurons ${ }^{77}$. In line with a driving effect of feedforward connections, microstimulation in the primary visual cortex (area V1) of monkeys activates neurons in higher area V4. By contrast, V4 microstimulation influences the V1 activity elicited by a visual stimulus, but has little influence in the absence of visual input, in accordance with a modulatory feedback effect $^{78}$.

\section{Neuromodulation}

All cortical layers receive neuromodulatory input from several deep brain nuclei. These systems include the dopaminergic system of the ventral tegmental area, the serotonergic dorsal and medial raphe nuclei, noradrenergic projections from the locus coeruleus and cholinergic afferents from the basal forebrain (Fig. 2). These modulatory systems provide information about the state of arousal and rewards and punishments and may influence synaptic transmission ${ }^{79}$ and cortical states ${ }^{80,81}$. In addition, they may play a part in learning by steering synaptic plasticity ${ }^{82-84}$ (discussed below).

\section{Cortical plasticity and learning}

Learning changes the response properties of neurons in many areas of the cerebral cortex $^{85}$ and subcortical structures ${ }^{86-88}$. Here we provide examples of studies on the effects of learning on neuronal tuning to stimuli in the visual ${ }^{89-91}$ and association cortices $^{92}$, demonstrating that neurons become tuned to feature variations that matter for a task.

In one study, Schoups et al. ${ }^{89}$ trained monkeys to perform an orientation discrimination task. The animals judged whether the orientation of a grating stimulus was rotated clockwise or anti-clockwise relative to a reference orientation (Fig. 3a). At the beginning of training, the monkeys needed an orientation difference of 10 degrees or more to be able to perform the task reliably. However, after months of training, they performed the task with orientation differences as small as 1 degree. As a result of training, V1 neurons became better in discriminating between small difference in orientation, an effect that was most pronounced for neurons with a preferred orientation that differed only slightly (for example, by about 15 degrees) from the trained orientation (Fig. 3b). For these neurons, the trained orientation fell on the highest-gradient part of the tuning curve, and training increased the gradient of that part (Fig. 3c). Exposure to task-irrelevant stimuli, presented at another location during task performance, did not cause comparable changes in neuronal tuning. Thus, the mere presentation of stimuli did not induce plasticity. 
Freedman and Assad ${ }^{92}$ reported related effects in the association cortex. They recorded the activity of neurons in area LIP of the parietal cortex of monkeys trained to categorize motion stimuli. The monkeys saw stimuli with dots moving in one of twelve directions. that were divided into two arbitrary categories (Fig. 3d) on either side of a 'category boundary'. On each trial, the monkeys first saw a sample stimulus and remembered its category so that they could report whether a later stimulus belonged to the same or the other category. Figure $3 e$ illustrates the tuning of an LIP neuron that responded more strongly to motion in all motion directions of one category (blue in Fig. 3d) than any of the directions of the other category (red in Fig. 3d). A comparison of responses to stimuli with adjacent motion directions revealed that the largest differences in firing rates were observed for pairs stimuli straddling the category boundary (Fig. 3f). Hence, learning to categorize stimuli causes increases in the sensitivity of neurons to category boundaries. These results raise a number of important questions.

The first question is about the connections that change during learning. In the orientation discrimination task, the sharpening of V1 tuning curves occurred in L2-3 and $L 5-6$ but not in L4, the input layer of cortex. This might suggest that connections from $L 4$ to the other layers undergo plasticity. However, other studies have demonstrated plasticity in the connectivity between sensory cortices ${ }^{93}$ and between the sensory cortex and subcortical structures ${ }^{86,88,94}$. In one study ${ }^{86}$, rats trained to distinguish between auditory tones with different pitches showed strengthened connections between the primary auditory cortex and the striatum. Another study in mice revealed that the connections between the visual cortex and the accessory optic system, which controls the gain of the optokinetic reflex [G], undergo plasticity after a lesion of the vestibulum ${ }^{88}$. Hence, plasticity of the connections within the cortical columns as observed by Schoups et al. (Fig. 3a-c) is complemented by plasticity of other connection types. It seems likely that the precise contributions of plasticity of these different connection types to learning depend on the task and they remain to be fully understood.

A second question is: how do neurons in sensory and association areas become tuned to a category boundary that can only be inferred by observing a reward structure - that is, contingent on the stimuli and choices across trials? A possible solution is that feedback connections from the response-selection stage assign credit (or blame) by tagging those synapses in sensory and association cortices that were responsible for action selection (that is, placing eligibility traces; Fig. 1). If an action is rewarded, the tagged connections are strengthened by a change in neuromodulator concentration that promotes synaptic potentiation (Fig. 1) to increase the probability that the same response reoccurs in the future. If the animal makes a wrong choice, feedback connections from neurons coding for this erroneous action tag another set of synapses, which decrease in strength owing to a change in the neuromodulator 
concentrations coupled to the lack of reward (Fig. 1). Such an interplay between feedback connections and neuromodulators (formalized by equation (3)) can explain the emergence of category selectivity in sensory and association cortices ${ }^{28,32,95}$ (Box $1)$.

A third question relates to the identity of the synaptic tags and their interaction with neuromodulatory systems. Usually there are delays between the activity in sensorimotor pathways and the moment when the organism can evaluate whether the outcome of a response was better or worse than expected ${ }^{11}$. Synaptic tags would have to persist long enough to bridge the delay. Below, we review initial insights into the molecular identity and persistence of tags and how they might interact with neuromodulatory systems.

\section{Gating and steering plasticity}

Now, we discuss the factors that influence plasticity, distinguishing between those that gate plasticity and those that steer plasticity. We propose that feedback signals from the response-selection stage gate plasticity by placing tags on the synapses that promoted selection of an action and that therefore should be held 'responsible' for the action outcome. By contrast, neuromodulators are proposed to steer plasticity by conveying the RPE, which is either positive and promotes synaptic potentiation, or negative, leading to synaptic depression ${ }^{19}$.

\section{Gating of synaptic plasticity}

Evidence for strong relations among action selection, selective attention and the influence of feedback connections on sensory cortices comes from psychology as well as neurophysiology. Psychological studies have demonstrated that every visually guided movement of the eye or the arm is associated with a shift of visual attention to the target of the movement ${ }^{26}$. Furthermore, neurophysiological experiments in non-human primates have demonstrated that when an animal plans a saccade to a visual object, neuronal activity elicited by this object in the visual and motor cortices is enhanced over the activity elicited by non-selected stimuli ${ }^{27,96}$. These response enhancements in the visual cortex are the neural correlate of a shift of attention towards the target of the subsequent eye movement.

The curve-tracing task provides a good illustration of the coupling between action selection and attention (Fig. 4). In this task, monkeys (or humans ${ }^{97}$ ) direct their gaze to a fixation point and a stimulus appears with a number of curves. One of the curves is a target curve and connects the fixation point to a larger circle, which is the target of a saccade. The monkeys must mentally trace the target curve to locate the saccade target in order to obtain a reward and must ignore other curves, which are distractors. The appearance of the curves activates neurons in many cortical areas, including V1 and the frontal eye fields [G]. The initial part of the response in each of 
these regions is dominated by feedforward processing, and does not distinguish between target and distractor curves (Fig. 4c). After this phase, the animal mentally traces the target curve, while maintaining gaze at the fixation point. Now, feedback connections and horizontal connections help enhancing the representation of the target curve in the visual and frontal cortex ${ }^{96}$ (Fig. 4b). The relative increase in neuronal activity caused by this mental tracing corresponds to the spreading of attention across the target curve ${ }^{98}$. If the monkey mistakenly selects the distractor curve and makes the saccade to the circle at the end of the distractor curve, the representation of the distractor curve is enhanced in the visual cortex ${ }^{96,99,100}$ (as in Fig. 1). Hence, the attentional feedback signals from frontal cortex enhance the activity of activated circuits in the association and sensory cortices that are accountable for the selected eye movement. Thus, they may enable ('gate') the plasticity of those connections that should change if the action outcome is better or worse than expected.

Feedback pathways could tag synapses for plasticity via two routes: via corticocortical feedback connections and/or through the thalamus. Both routes target distal dendrites in the superficial layers and L5 (Fig. 2). In monkeys, selective attention increases the activity not only of neurons in the visual cortex ${ }^{101,102}$ but also in the pulvinar, a higher-order visual thalamic nucleus ${ }^{103}$ (equivalent to the lateral posterior thalamic nucleus in rodents). Inactivation of the pulvinar decreases visually driven cortical activity ${ }^{104}$, and impairs performance in tasks that demand attention shifts ${ }^{103-105}$. Furthermore, pulvinar lesions interfere with new learning ${ }^{106}$.

In support of the gating hypothesis, one study ${ }^{77}$ in mice demonstrated that activity in a higher-order thalamic nucleus indeed feeds back to sensory cortex to gate plasticity. The researchers recorded from the primary somatosensory cortex (S1) and investigated the plasticity of the connections that convey sensory information from the whiskers through the ventral posteromedial nucleus (VPm), the primary sensory thalamic nucleus. Repetitive stimulation of the whiskers induced long-term potentiation (LTP) in L2/3 pyramidal cells. Interestingly, LTP induction depended on activity in the posterior medial nucleus (POm), a cluster of higher-order nuclei in the somatosensory thalamus. Exogenously evoked POm neuron activity induced longlasting (>150 ms), NMDAR-dependent plateau potentials, probably caused by calcium influx, in the distal dendrites of the L2/3 neurons in S1. Notably, LTP of the L2/3 pyramidal cell response to whisker stimulation only occurred if the feedforward input coincided with the L2/3 plateau potentials in S1; blocking POm activity with muscimol decreased the S1 plateau potentials and abolished S1 LTP. LTP could also be blocked by injection of NMDAR antagonists into S1, which also blocked calcium influx into the distal dendrites (Fig. 5). Thus, feedback-mediated NMDAR-dependent plateau potentials are apparently necessary for making synapses that are activated by the excitatory feedforward pathway eligible for plasticity. 
POm neurons in rodents become active when ascending driving inputs from the sensory brainstem coincide with descending driving inputs from $\mathrm{L} 5$ neurons in $\mathrm{S} 1^{68,69}$, but whether POm conveys information about the selected action to S1 remains to be clarified. More is known about the visual modality in monkeys, in which selective attention activates the pulvinar and may specifically tag cortical synapses responsible for the selected action, given the relation between attention and action selection mentioned above.

Other studies in mice have demonstrated direct effects of corticocortical feedback connections from the primary motor cortex (M1) on S1, by examining different phases in the activation of S1 neurons by a tactile stimulus (just as the phases of V1 responses in the curve-tracing task). The early phase of the S1 response is driven by the feedforward sensory input, whereas later activity also depends on feedback from higher cortical areas, including $\mathrm{M} 1^{107}$, which cause plateau potentials and calcium influx into the apical dendrites of $L 5$ neurons ${ }^{107,108}$. Interestingly, late-phase S1 activity $^{109}$ and calcium influx into S1 dendrites predict the reporting of the sensory stimulus by the animal (by licking) ${ }^{110}$, in support of the idea that action selection in motor cortex causes upstream effects in sensory cortex (Fig. 5c). Moreover, pharmacological or optogenetic suppression of the late activity ${ }^{109}$ or of plateau potentials in $\mathrm{S} 1^{110}$ impairs the licking response, in particular for weak tactile stimuli. It remains to be determined, however, whether the M1 feedback also gates plasticity in $\mathrm{S} 1$, as does feedback from the POm ${ }^{77}$.

Gating of plasticity may also depend on disinhibitory circuits ${ }^{111}$ in the cortical column that involve VIP-positive interneurons ${ }^{60,112,113}$. These VIP ${ }^{+}$neurons receive input from multiple sources, including feedback from higher-order thalamic nuclei ${ }^{114-116}$, and inhibit somatostatin (SST)-positive interneurons that, in turn, inhibit the activity of pyramidal neurons ${ }^{59,117-119}$. SST $^{+}$neurons largely overlap with Martinotti cells [G], which inhibit activity in the distal dendrites of pyramidal neurons ${ }^{120}$, near the synapses formed by feedback connections from higher-order thalamic nuclei ${ }^{121-123}$. When VIP+ neurons suppress the activity of $\mathrm{SST}^{+}$neurons, they may thereby enable the influx of calcium into these distal dendrites and thus 'switch on' synaptic plasticity ${ }^{124-127}$. Indeed, in mice, the optogenetic inhibition of $\mathrm{SST}^{+}$neurons enhances V1 plasticity induced by the closure of one eye $\mathrm{e}^{126}$. Furthermore, the optogenetic or chemogenetic silencing of $\mathrm{SST}^{+}$neurons as well as their deletion promotes learningdriven plasticity in $\mathrm{M} 1^{124,125}$.

It seems likely that the effects of feedback on plateau potentials, sensory perception and plasticity observed in S1 of mice generalize to other sensory modalities and other species. Synaptic plasticity in the mouse hippocampus was recently shown to depend on plateau potentials ${ }^{128}$ and to be sculpted by inhibition ${ }^{129}$. In mouse V1, NMDAR-dependent calcium events in dendrites enhance the stimulus selectivity of neurons ${ }^{130}$. Furthermore, in monkeys, feedback connections to V1 target the 
superficial layers and L5 and activate NMDARs to increase the representation of stimuli that matter for behaviour ${ }^{74}$.

The data reviewed above suggest that response selection elicits feedback signals that enable the plasticity of upstream synapses. This gating hypothesis provides possible mechanisms that may explain the psychological finding that animals learn what they attend. Although we focused on reinforcement learning, it is conceivable that attention and feedback connections have equivalent roles in forms of unsupervised learning [G], where behavioural outcomes are not crucial ${ }^{77,131-133}$. For example, the learning of abstract visual concepts such as birds or cars relies on similar interactions between lower visual brain regions coding for primitive features and higher areas coding for semantic categories. That is, during unsupervised learning, neurons in higher areas could feed back to gate the synaptic plasticity of relevant low-level feature representations.

\section{Steering of synaptic plasticity}

The RPE should steer plasticity; that is, it should determine whether the tagged synapses undergo potentiation or depression. A widely held hypothesis is that the RPE is signalled by released neuromodulators. We briefly review the possible influence of dopaminergic, cholinergic, serotonergic and noradrenergic projections on cortical plasticity, but we note that other neuromodulatory systems, such as histamine signalling ${ }^{134}$ and neuropeptide signalling ${ }^{84}$ may also have a role. In addition to their role in coding the RPE, neuromodulator levels may also signal other behavioral states, including novelty, surprise, arousal and emotional valence ${ }^{17,18}$. These factors may also influence plasticity through effects on the release of neuromodulators.

Dopamine. The ventral tegmental area is the main source of dopamine for the cortex. Many, but not all, dopamine neurons are active if an animal receives more reward than it expected ${ }^{135-137}$. Dopamine projections target subcortical structures, including the striatum, as well as the cortex, where the projections are densest in prefrontal and motor cortices and sparser in sensory areas. Dopaminergic signalling occurs through five metabotropic receptor subtypes, of which the D1 receptor (D1R) is the most abundant in the cortex. D1R ultimately activates protein kinase A (PKA), which is strongly implicated in long-term plasticity. Furthermore, dopamine may modulate synaptic release and the incorporation of AMPARs and NMDARs into the cell membrane ${ }^{79}$. Dopamine regulates synaptic plasticity in the striatum ${ }^{14}$, hippocampus ${ }^{17}$ and also in the auditory cortex, where the pairing of a particular tone with electrical stimulation of the ventral tegmental area causes an expansion of the cortical area representing the tone frequency ${ }^{138}$. Many dopamine neurons code for RPEs and are in a position to steer plasticity in structures containing dopamine 
receptors. Other neurons in the ventral tegmental area code for motivational signals besides the RPE and may thus also play a part in steering plasticity ${ }^{17,139}$.

Acetylcholine. Cholinergic signalling in the neocortex is thought to have an important role in the control of brain states, attention and learning. It is highly upregulated during wakefulness and with sustained attention ${ }^{80}$. Cholinergic projections from the basal forebrain are widely distributed in the cortex and show a complex topographical, modality-related organization ${ }^{140,141}$. The effects of acetylcholine in the cortex are mediated by metabotropic muscarinic receptors and ionotropic nicotinic receptors. Nicotinic receptors are expressed presynaptically on some thalamocortical axons ${ }^{142}$ and postsynaptically on $\mathrm{VIP}^{+}$interneurons that also express the ionotropic serotonin receptor ${ }^{114,143}$. Muscarinic receptors are expressed both presynaptically and postsynaptically by pyramidal cells, where they can have mixed effects $^{80}$. Optogenetic activation of cholinergic projections in mice enhances visual responsiveness of neurons in V1 and improves performance in an orientation discrimination task ${ }^{144}$. Many cholinergic neurons respond to punishment, and a smaller number also respond to unexpected rewards, compatible with a role in RPE signalling ${ }^{145,146}$. Nevertheless, other behavioural factors, such as arousal level, could also influence plasticity because they are associated with changes in acetylcholine release. Electrical stimulation of cholinergic centres enhances plasticity in the visual cortex of mice and the auditory cortex of mice and rats ${ }^{147-151}$, whereas the depletion of acetylcholine suppresses synaptic plasticity in the auditory and somatosensory cortex of rats ${ }^{151,152}$. Accordingly, pharmacological blockers of cholinergic signalling, or the depletion of cholinergic fibres to the temporal lobe using toxins, impairs recognition memory and the learning of new sensory stimuli ${ }^{153,154}$ and lesions of the cholinergic nuclei impair spatial learning ${ }^{155}$. These results, taken together, indicate that cholinergic neurons could steer cortical plasticity.

Serotonin. The serotonergic system is thought to modulate sensory processing, cognition and emotional states, and to regulate innate behaviours such as food intake and reproduction ${ }^{156}$. Serotonergic projections to almost all regions of the forebrain originate from two rostral serotonergic clusters in the brainstem: the median and dorsal raphe nuclei (MRN and DRN) ${ }^{156}$. In the cortex, the effects of serotonin are highly diverse, and mediated by a vast repertoire of presynaptic and postsynaptic metabotropic and ionotropic receptors ${ }^{83,157}$. Among other factors ${ }^{156}$, the activity of serotonergic neurons depends on the amount of reward or punishment that is anticipated and received ${ }^{158-163}$; however, the effects of rewardrelated serotonergic signalling in cortex have remained unclear. The activation of cortical serotonergic inputs facilitates the delivery of AMPARs to synapses ${ }^{82,83}$, and sharpens the whisker barrel map of rats during visual deprivation ${ }^{164}$. Thus, serotonin also affects cortical synaptic plasticity. 
470 Noradrenaline. Noradrenergic signalling is associated with arousal ${ }^{165}$ and with the

471 receipt of rewarding stimuli ${ }^{166}$. The most important source of noradrenaline is the

472 locus coeruleus, which projects widely to all other neuromodulatory centres, as well

473 as to all regions and layers of the cortex. Activity of the locus coeruleus affects

474 various cognitive and sensory processes ${ }^{165}$. For example, increased activity of the

475 locus coeruleus enhances sensory-evoked responses in the thalamus and

476 cortex ${ }^{167,168}$. Noradrenaline exerts its effects predominantly through

477 adrenoreceptors, which influence synaptic plasticity ${ }^{82,169}$. Furthermore,

478 noradrenergic signaling has been shown to induce plasticity in the hippocampus,

479 amygdala and neocortex of rodents, and to enhance contextual learning ${ }^{170}$, fear

480 conditioning ${ }^{171}$ and auditory perception ${ }^{167}$.

481

482 Spike-timing-dependent plasticity. Theories about the implementation of

483 reinforcement learning in the brain have proposed that the global release of the

484 neuromodulators influences plasticity in order to determine whether selected

485 actions will be taken again in the future ${ }^{16,19}$. They can do so by modifying synapses

486 (for example, by changing the surface expression of receptors [Au:OK?]) or by

487 changing the intrinsic properties of neurons ${ }^{11,19,25,28,169,172}$. Several studies have

488 examined the influence of different neuromodulators on spike-timing-dependent

489 plasticity [G] (STDP), wherein the increase or decrease of synaptic strength depends

490 on the precise time interval between presynaptic and postsynaptic action potentials.

491 These studies demonstrated that dopamine, acetylcholine, noradrenaline, serotonin,

492 but also endocannabinoids, may increase or decrease the sensitivity of neurons to

493 STDP paradigms, can modify the shape of the STDP function and can even determine

494 whether synapses undergo potentiation or depression ${ }^{14,84,132,169,173-175}$. Thus,

495 substantial evidence indicates that neuromodulatory systems steer neuronal

496 plasticity. However, the field has yet to reach a consensus about the relative

497 importance of these neuromodulatory systems - alone or in their combination -

498 and their precise roles in the control of plasticity.

\section{Gating and steering together}

501 The combination of corticocortical or thalamocortical feedback connections and

502 neuromodulatory signals can ensure that the information necessary for the synaptic

503 update becomes available locally, at the synapse undergoing plasticity (Box 1). This

504 can be illustrated for an example reinforcement learning scenario (Fig. 6a). First,

505 activity propagates from sensory cortex to motor cortex, and the selected motor

506 programme provides feedback to earlier processing levels. Coincident activity of

507 feedforward and feedback pathways specifically occurs in the cortical columns that

508 will be held accountable (Fig. 6a). In these columns, corticocortical and

509 thalamocortical feedback connections induce calcium events in pyramidal dendrites, 
510 either through direct excitation or through indirect VIP+-neuron-mediated

511 disinhibition. These events induce eligibility traces at the activated feedforward

512 synapses - that is, biochemical modifications that enable their plasticity. One or a

513 few seconds later, the action outcome is evaluated and an RPE computed, which

514 then steers the plasticity. Eligible synapses are potentiated by neuromodulators if

515 the RPE is positive (as shown in Fig. 6a), and weakened if the RPE is negative. The

516 release of neuromodulators can be separated in time from the activation of the

517 neurons, because the tags can persist in the absence of neuronal spiking ${ }^{14,174,175}$.

518 Indeed, the persistence of eligibility traces may be related to longer-term

519 interactions between plasticity-inducing events that have been observed in the

520 hippocampus and gave rise to the 'synaptic tagging and capture hypothesis' $15,176,177$.

521 According to this hypothesis, weak plasticity-inducing events induce synaptic tags

522 that cause these synapses to undergo plasticity if stronger plasticity-inducing events

523 occur at other synapses of the same neuron within hours. As such, the strong

524 potentiation of the other synapses cause the production of plasticity-related

525 proteins, which are captured by tagged synapses so that they too change their

526 strength. The hypotheses that synaptic tags interact with plasticity-related

527 proteins ${ }^{15,176}$ or with neuromodulators coding for the RPE ${ }^{11,32}$ are not mutually

528 exclusive and occur at different time-scales: that is, seconds to bridge delays in

529 reinforcement learning and hours for synaptic tagging and capture. Future research

530 could aim to better characterize the processes that act on synaptic tags to control

531 plasticity.

532 Although we focus above on the role of neuromodulatory inputs in steering

533 plasticity, some studies indicate that neuromodulators may also participate in gating

534 processes by altering neuronal excitability ${ }^{178}$ - for example, by altering presynaptic

535 glutamate release ${ }^{179}$ or by activating disinhibitory circuits $60,84,112,113,117,118$. It is

536 important to note, however, that the neuromodulatory projections are relatively

537 diffuse, which implies that any gating function they have is likely to be less specific

538 than that of corticocortical and thalamocortical feedback connections, which are

539 better positioned to tag specific relevant synapses.

540 In line with the ideas presented above, a recent study documented the existence of

541 synaptic tags that make synapses eligible for plasticity and that are influenced by the

542 later release of neuromodulatory substances in the striatum ${ }^{14,175}$. Yagishita et al. ${ }^{14}$

543 activated a single spine of neurons in slices by uncaging glutamate, while causing the

544 same cells to fire action potentials by injecting current. If dopamine was released

545 within a time window of $\sim 1 \mathrm{~s}$ after this event, the volume of the activated spine

546 increased. This potentiation depended on the activity of NMDARs and several

547 intracellular messengers and on the delayed signalling in a pathway initiated by the

548 binding of dopamine on D1Rs (Fig. 6b). These two pathways downstream of NMDARs

549 and D1Rs converge to activate CaMKII, which is most active when dopamine is 
released after co-activation of the presynapse and postsynapse. Similar interactions between NMDAR-dependent plasticity and delayed dopamine availability occur in hippocampal slices, with intervals in the minute range ${ }^{180}$. It is not yet known if comparable interactions take place in the synapses of cortical neurons, although this could be tested with current technology. The mechanisms at work within the synapses are complex and we therefore expect that many discoveries about the interaction between glutamatergic transmission and neuromodulatory signals are still to be made. These studies could give new insight into how attentional feedback signals and RPEs interact to optimize the contribution of synapses to behaviour.

\section{Conclusions}

In recent years, researchers have made substantial progress in understanding how the neural circuits of the brain are rewired as the result of learning. Above, we have focused on the malleability of representations in sensory and association cortex and reviewed evidence for a role of corticocortical and thalamocortical feedback connections on the one hand, and neuromodulatory influences on the other. In combination, these factors may permit learning rules that can train the cortical circuitry to refine the representations of sensory stimuli as well as their mapping on to appropriate motor responses. The resulting learning rules can be implemented by synapses in the brain to overcome the credit assignment problem. We have briefly touched on the emerging insights into how gating and steering factors impact on the biochemical cascades that control whether a synapse strengthens or weakens. Future studies could test whether corticocortical and thalamocortical feedback tags the circuits that are responsible for stimulus-response mapping for plasticity, and could elucidate the identity of the tags and how they make synapses susceptible for neuromodulatory signals. Although feedback connections seem to enable the plasticity of feedforward connections, it remains to be determined whether the interactions between feedforward and feedback connections take place with cellular precision or with courser resolution at the level of, for example, the cortical column. Furthermore, future studies could examine how gating and steering factors might work together in scenarios besides reinforcement learning, considering roles of feedback connections and neuromodulatory systems in, for example, the detection of novelty and surprise.

Although many of the processes determining synaptic plasticity remain to be discovered, it is encouraging that we have reached a stage where insights from molecular, cellular and systems neuroscience and from theories of reinforcement learning and deep artificial networks inform each other and may now be integrated into a unified framework for learning in the brain.

\section{Acknowledgements}

The authors thank S. Bohte, C. Pennartz, M. Sherman, V. Kehayas, and H. Kennedy 
591 for helpful input and comments. The work was supported by the Netherlands

592 Organisation for Scientific Research (NWO; ALW grant 823-02-010 to P.R.R.), the EU

593 Seventh Framework Programme (grant agreement 7202070 'Human Brain Project'

594 to P.R.R., and European Research Council (ERC) grant agreement 339490

595 "Cortic_al_gorithms" to P.R.R.), the Swiss National Science Foundation (SNF;

596 research grants 31003A-153448, CRSII3-154453 to A.H., and the National Centre

597 Competence in Research (NCCR) Synapsy grant 51NF40-158776 to A.H.), and the

598 International Foundation for Research in Paraplegia (to A.H.).

599

\section{Author contributions}

601 P.R.R. and A.H. researched data for the article, made substantial contributions to

602 discussions of the content, wrote the article and reviewed and/or edited the

603 manuscript before submission.

604

605 Competing interests

606 The authors declare no competing interests.

607 Publisher's note

608 Springer Nature remains neutral with regard to jurisdictional claims in published 609 maps and institutional affiliations.

\section{Box 1 | Deep learning in the brain}

613 In recent years, great advances have been made with deep artificial neural networks

614 that are composed of many layers and that are trained with the so-called error-

615 backpropagation rule, a method that specifies how connections between the the

616 units of a network should change during training. The error backpropagation adjusts

617 synaptic weights in networks that are composed of several layers to reduce the

618 errors in the mapping of inputs into the lower layer to outputs in the top layer. It

619 does so by first computing the error, which is the difference between the actual and

620 desired activity levels of output units. Error backpropagation then determines how

621 the strength of connections between successively lower layers should change to

622 decrease this error, by computing derivatives [G] using a method known as gradient

623 descent $[\mathrm{G}]^{3}$. Artificial neural networks trained by error backpropagation now attain

624 human-level performance in image recognition ${ }^{4}$ and in some computer games ${ }^{33}$.

626 Artificial image-recognition systems usually take a convolutional network approach,

627 in which the complexity of tuning of units increases in higher layers, and specialized

628 layers are interspersed to pool activity across space and to build receptive fields that

629 are translation-invariant [G] (see the figure below). The tuning of units at lower and

630 higher levels in these convolutional networks resembles the tuning of neurons in

631 lower and higher areas of monkeys and humans ${ }^{38,181}$. In convolutional networks, 
632

633

634

635

636

637

638

640

642

643

644

645

646

647

648

649

650

651

652

653

654

655

656

657

658

659

660

661

662

663

664

665

666

667

668

669

670

671

many weights are shared - that is, copied from one location in the network to another - which is biologically implausible. Furthermore, back in 1989 Francis Crick argued that the error-backpropagation rule itself is neurobiologically unrealistic ${ }^{34}$. He found it difficult to imagine how synapses in the brain could determine the change in their strength that would decrease the overall network error -; that is, how they could compute their own local error derivative.

However, researchers have proposed new ways in which learning rules that are equivalent to error-backpropagation might be implemented in the brain ${ }^{28,32,95,182-184}$ (reviewed elsewhere ${ }^{185}$ ). Specifically, learning rules such as AGREL (attention-gated reinforcement learning) ${ }^{28}$ and AuGMEnT (attention-gated memory tagging $)^{32}$ explain how synapses in deep networks can change to optimize reward outcome during reinforcement learning, in a biologically realistic manner. As the equations that establish the relation between these new learning rules and error backpropagation are somewhat complex, we refer the mathematically inclined reader to the original publications ${ }^{28,32}$. Conceptually, the main insight is that the synaptic error derivative can be split into two factors: first, the steering RPE that codes for the global network error and reaches all synapses through the release of neuromodulators, and second, a gating signal from the response-selection stage and carried by feedback connections that indicates how much of the credit or blame should be attributed to the individual synapse. These steering and gating factors jointly determine synaptic plasticity (as in equation (3) of the main text). In AGREL and AuGMEnT, the strength of feedback connections becomes proportional to that of feedforward connections during learning, and thus the learning rules become computationally equivalent to error backpropagation. Interestingly, approximate reciprocity between feedforward and feedback connections and efficient learning can also emerge if feedback connections are fixed and only feedforward connections are plastic, through a process called feedback alignment [G] ${ }^{31}$.

In other words, the brain can solve the credit-assignment problem in a manner that is equivalent to deep learning. Accordingly, these rules can be used to train simple artificial neural networks on several tasks that monkeys can be trained on by trial and error ${ }^{32}$, and their capability goes beyond biologically plausible learning rules that do not feature plasticity-gating feedback connections. Interestingly, these networks make many of the mistakes that are also made by animals undergoing training, and the tuning of units at intermediate network levels becomes similar to that of neurons in the visual and association cortex ${ }^{13,28,32}$ (leading to tuning curves similar to those seen in trained animals, such as those in Fig. $3 c, f)$. Hence, developments in many disciplines - from molecular biology to machine learning and cognition - may now pave the way for a genuine understanding of how deep learning is implemented in the brain.

\section{Figure Legends}


673

674

675

676

677

678

679

680

681

682

683

684

685

686

687

688

689

690

691

692

693

694

695

696

697

698

699

700

701

702

703

704

705

706

707

708

709

710

711

712
Figure 1 | Putative control signals that influence synaptic plasticity. During sensory processing, feedforward connections (black connections) propagate activity from lower to higher areas (left side of figure). Neurons in frontal cortex compete to determine the selected action. If an action has been selected, the 'winning' neurons provide an attentional feedback signal to the lower-level synapses responsible for the selected action (red connections), enabling their plasticity in a process that may be related to calcium events in dendrites (middle part of the figure). This enabling is called 'tagging' (red Ts represent tagged connections). The other connections are not plastic (dashed connections). Note that different actions enable plasticity of different connections as illustrated (different rows of the network). Neuromodulators code for the reward-prediction error (RPE) - that is, whether the outcome was better (green) or worse (red) than expected, and determine whether the tagged synapses increase (thicker connections) or decrease in strength (thinner connections). The lower panel is adapted from REF ${ }^{25}$.

\section{Figure 2 | Cortical feedforward, feedback and neuromodulatory information}

streams. Diagram of intracortical (a), long-range (b), subcortical (c) and neuromodulatory (d) connections within, to and from the sensory and association cortices. The main axodendritic synaptic input patterns are shown as arrows. a | Intracortical information streams include local interactions within and between cortical columns. Input to L4 and L2/3 propagates to all other layers (except L1) through ascending and descending connections. Horizontal connections distribute signals within $L 2 / 3$ and $L 5 a$, whereas feedback is provided from $L 6$ and $L 2 / 3$ to $L 4$, and from $L 5 a$ to $L 2 / 3$. b,c | Information exchange between cortical areas occurs through long-range corticocortical connections and transthalamic pathways. Firstorder (FO) thalamus provides input to lower cortical areas (level I in c). Cortical L5 output reaches higher-order $(\mathrm{HO})$ thalamus, which in turn feeds forward to higher cortical areas (level II in c) or back to lower-order cortex (level I). Feedforward and feedback streams are segregated in different layers, to great extent in primates, and to some extent in rodents ${ }^{45,186}$. In primates, neurons in the deeper $L 3$ and the superficial L5 project forward to $L 4$ of higher-order cortical areas. Neurons in superficial $L 2 / 3$ and in L5/6 of higher areas send feedback projections to $L 1$ and $L 5$ of lower areas ${ }^{43,56}$. In rodents, separate feedforward and feedback projections may originate from molecularly distinct neuronal subtypes ${ }^{45}$, but their distribution across the lamina is 'salt-and-pepper'-like ${ }^{186,187}$. L1 is a main feedback layer, where inputs impinge on apical dendrites of pyramidal neurons. $\mathbf{d}$ | Patterns of neuromodulatory input to cortex remain poorly characterized. The current view holds that virtually all types of neuromodulation arrive in all layers of all cortical areas ${ }^{82}$, although some topographic organization and laminar specificity is observed for the cholinergic 
projections ${ }^{140,141}$. Neuromodulatory signalling occurs via both synaptic transmission and volume transmission, and in most instances through metabotropic receptors ${ }^{82,188}$. The diagram is a composite, adapted from refs. ${ }^{37,39,40,43,45,46}$.

Figure 3 | Effects of learning on neuronal tuning curves. a | Monkeys in the study by Schoups et al. ${ }^{89}$ judged whether the orientation of the lower grating was tilted clockwise or anticlockwise from a right oblique orientation (green line, 45 degrees). They could always ignore the upper grating, as it was a distractor. The small circle on the left denotes a fixation point. b | Orientation tuning curves of example V1 neurons (green arrow indicates the trained orientation). Thick line segments highlight the slope of the tuning curves at the trained orientation. c | The slope of V1 neuron tuning curves at the trained orientation, as a function of the neurons' preferred orientation (percent change in firing rate per degree of orientation). Training in the orientation judgement task increased the slope of tuning curve of neurons whose preferred orientation differed only slightly (by $\sim 16^{\circ}$ ) from the trained orientation, and that were maximally informative for the task. The blue dashed line shows the slope of the tuning curves before training, whereas the red line shows the slope after training. $\mathbf{d}$ | In a study by Freedman and Assad ${ }^{92}$, monkeys saw dots moving in one of 12 directions that were divided into two categories (red and blue arrows). The animals compared the category of a sample stimulus (cue-1) to that of a later probe stimulus (cue-2) and released a lever if the categories were the same. e | Activity elicited by the sample directions in an example LIP neuron. The neuron gave similar responses for stimuli of the same category (red or blue) but there were larger differences in activity between stimulus categories. $\mathbf{f}$ | Distribution of adjacent motion directions giving rise to the largest difference in stimulus-driven activity of individual LIP neurons. Note that for most cells, the largest changes in activity occurred at the category boundaries. Parts a-c are adapted from REF. ${ }^{89}$. Parts $d-f$ are adapted from REF. ${ }^{92}$.

\section{Figure 4 | Attentional selection and eye movement selection during curve-tracing.}

a | The animal first directs gaze to a small fixation point (FP). After a short delay, two curves appear on the screen. The curve that is connected to the fixation point is the target curve $(T)$, and the other curve is a distractor (D). After an additional delay, the FP disappears, and the monkey makes an eye movement to the larger red circle that was previously connected to the fixation point (that is, the end of the T curve). $\mathbf{b}$ | In the left panel, the receptive fields of neurons in V1, V4 and fall on the target curve, whereas in the middle panel, they fall on the distractor curve. c | During an initial feedforward processing phase (black bars), neurons in areas V1 and FEF are activated by the appearance of a curve in their receptive field (dashed line at time zero). In a later, recurrent processing phase, feedback connections come into play 
753

754

755

756

757

758

759

760

761

762

763

764

765

766

767

768

769

770

771

772

773

774

775

776

777

778

779

780

781

782

783

784

785

786

787

788

789

790

791

792

(red bars) and now the representation of the target curve that is selected for an eye movement response is enhanced (red lines) in both brain regions, compared to the representation of the non-selected distractor (blue dashed lines). Part $\mathbf{c}$ is reproduced from ref. ${ }^{100}$.

\section{Figure 5 | Gating of plasticity of feedforward connections to S1 by higher-order} thalamic nuclei. a | Schematic of the experiment; somatosensory thalamocortical and corticothalamic pathways. Whisker stimulation-driven sensory postsynaptic potentials, and the potentiation thereof, in L2/3 neurons were assessed using wholecell recordings in vivo. A sensory stimulus applied to the whisker activates feedforward inputs (from the VPm) and feedback inputs (from the POm) to S1, which causes NMDAR-mediated potentials in pyramidal cells. Activity of POm is also gated by input from other cortical areas and the zona incerta. $\mathbf{b} \mid$ Whisker deflections induce postsynaptic potentials in L2/3 S1 neurons that consist of two components: a

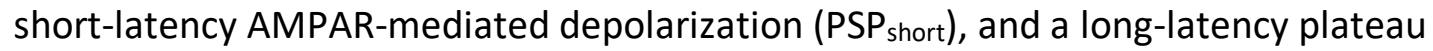

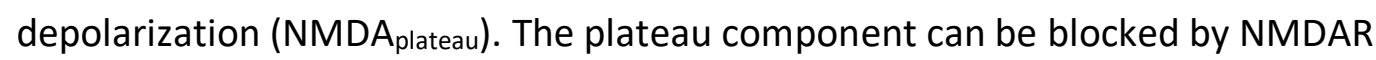
blockers (DAP5) as well as by muscimol injections targeted to the POm (top graph). These two methods of blocking of NMDAR-mediated plateau potentials prevent whisker deflection-induced synaptic potentiation (bottom graph). c | Schematic of a whisker-stimulus detection task and the imaging of calcium events in two pyramidal cell dendrites (\#1 and \#2) in S1. Upon weak whisker deflections near the detection threshold $(\theta)$, dendritic $\mathrm{Ca}^{2+}$ events are stronger in hit trials (stimulus detection, rewarded) trials than in miss trials (failed stimulus detection, unrewarded), suggesting that hit-related feedback inputs are involved in generating them. Parts a and $\mathbf{b}$ are adapted from ref. ${ }^{77}$, the mouse drawings in parts a and $\mathrm{c}$ are from ref. ${ }^{189}$ and the schematic and data in c are adapted and reproduced, respectively from ref. 110 .

Figure 6 | Gating and steering of synaptic plasticity. a | Within the cortical column, feedback connections (FB) target distal dendrites as well as disinhibitory circuits that enable plasticity (part (i)). In diagram (ii), the feedforward connections propagate activity to higher levels, which in turn provide feedback to the thalamocortical synapse that is going to be held responsible for the outcome of the action. The feedback does this by causing dendritic calcium events, which induce synaptic tags on activated thalamocortical synapses (and possibly other synapses in the same column). Diagram (iii), the tag remains once the activity of the column ceases. Diagram (iv), the reward prediction error gives rise to the release of neuromodulators to increase or decrease the strength of tagged synapses, influencing the probability that the same action will be selected in the future. Diagram (v), the tagged synapse has now been strengthened. $\mathbf{b}$ | Sequence of 
molecular events in postsynaptic spines in the striatum. The binding of glutamate to NMDARs gates plasticity through calcium influx. Neuromodulators, such as dopamine, activate another pathway through, for example, D1Rs, adenyl cyclase, CAMP (which is broken down by phosphodiesterase (PDE)) and PKA-DARPP32-PP1 signalling. Both pathways need to be active for the activation of CaMKII, which causes an increase in synaptic strength as measured by an increase in the volume of dendritic spines. Part $\mathbf{b}$ is reproduced from ref. ${ }^{14}$.

\section{Glossary}

\section{Reinforcement learning}

Trial and error learning while interacting with an environment and experiencing rewards and punishments as consequences of the chosen actions.

\section{Reward-prediction error (RPE)}

Difference between the amount of reward that was expected and the amount that was obtained

\section{Eligibility traces}

In reinforcement learning theory, eligibility traces are parameters that determine whether connections in a network undergo plasticity upon reward-prediction errors

\section{Synaptic tags}

Biochemical signals at synapses that determines whether they will undergo plasticity

\section{Error-backpropagation rule}

A mathematical method used to calculate the contribution of connections to the error of a network with multiple layers between input and output.

\section{Optokinetic reflex}

Innate reflexive smooth eye-movements elicited by large moving visual stimuli.

\section{Frontal eye fields}

Area of the frontal cortex involved in the planning of eye movements

\section{Martinotti cells}

Somatostatin-expressing inhibitory interneurons with a characteristic morphology that target the dendritic tufts of pyramidal cells in various cortical layers.

\section{Unsupervised learning}

Inferring the structure of unlabeled data when information about desired categorizations is not provided

\section{Spike-timing-dependent plasticity}


Plasticity rule where the change in the strength of synapses depends on the relative timing of pre- and postsynaptic action potentials

\section{Derivatives}

The derivative of the error function to a synaptic weight is the rate of change of the error when changing the strength of a particular synapse

\section{Gradient descent}

Mathematical optimization method that determines the direction of the vector of changes in all synaptic weights causing the largest decrease in the network's error

\section{Translation-invariant}

Independence of object-recognition of the object's location relative to the viewer.

\section{Feedback alignment}

If the feedback and feedback weights of a neural network are not reciprocal, errorbackpropagation causes feedforward weights to align, that is, to become more symmetrical.

\section{Key points}

1) In addition to pre- and postsynaptic mechanisms, synaptic plasticity depends on neuromodulatory substances and feedback connections from higher-order cortical and thalamic brain regions.

2) Synaptic plasticity in the brain depends on the reward-predication errors and on selective attention. Neuromodulatory systems code for the reward-prediction errors and feedback connections from the response selection stage mediate top-down attention effects.

\section{3) The combined influence of feedback connections and neuromodulatory} substances on plasticity enables powerful learning rules for the training of "deep", multilayered neuronal networks.
4) Feedback connections project to cortical layers that are distinct from feedforward input, where they impinge on distal dendritic segments, separate excitatory neuronal populations, or inhibitory interneurons.

5) Feedback connections gate plasticity in cortical pyramidal neurons by promoting NMDA-receptor driven calcium entry into dendrites and by disinhibition of the cortical column, among others by activating VIP-positive interneurons 
6) Synaptic tags are biochemical processes that make synapses eligible for plasticity.

880 Neuromodulators released later can interact with tagged synapses to increase or

881 decrease synaptic strength.

[Au: Please provide a list of up to 6 brief bullet points, each no more than 2 sentences long, highlighting the take-home messages of the Review.]

[Au: Highlighted references: For references that are particularly worth reading ( 10

\section{References}

892 1. Sutton, R. S. \& Barto, A. G. Reinforcement learning. (MIT Press, 1998).

8932 2. Littman, M. L. Reinforcement learning improves behaviour from evaluative feedback. Nature 521, 445-451 (2015).

3. Rumelhart, D. E., Hinton, G. E. \& Williams, R. J. in Parallel Distributed Processing: Explorations in the Microstructure of Cognition (eds. Rumelhart, D. E. \& McClelland, J. L.) 318-364 (MIT Press, 1986).

4. LeCun, Y., Bengio, Y. \& Hinton, G. Deep learning. Nature 521, 436-444 (2015).

5. Hebb, D. O. The organization of behavior. A neuropsychological theory. (1949).

6. Martin, S. J., Grimwood, P. D. \& Morris, R. G. M. Synaptic plasticity and memory: an evaluation of the hypothesis. Annu. Rev. Neurosci. 23, 649-711 (2000).

7. Schultz, W. Getting formal with dopamine and reward. Neuron 36, 241-263 (2002).

8. Niv, Y. \& Schoenbaum, G. Dialogues on prediction errors. Trends Cogn. Sci. 12, 265-272 (2008).

9. Baxter, J. \& Bartlett, P. L. Infinite-horizon policy-gradient estimation. J. Artif. Intell. Res. 15, 319-350 (2001).

10. Frémaux, N., Sprekeler, H. \& Gerstner, W. Reinforcement learning using a continuous time actor-critic framework with spiking neurons. PLoS Comput. Biol. 9, e1003024 (2013).

11. Izhikevich, E. M. Solving the distal reward problem through linkage of STDP and dopamine signalling. Cereb.Cortex 17, 2443-2452 (2007).

12. Legenstein, R., Pecevski, D. \& Maass, W. A learning theory for reward-modulated spiketiming-dependent plasticity with application in biofeedback. PloS Comput.Biol. 4, e100180(2008).

13. Rombouts, J. O., Bohte, S. M., Martinez-trujillo, J., Roelfsema, P. R. \& Pieter, R. A learning rule that explains how rewards teach attention. Vis. cogn. 23, 1-27 (2015).

14. Yagishita, S. et al. A critical time window for dopamine actions on the structural plasticity of dendritic spines. Science (80-. ). 345, 1616-1620 (2014).

15. Frey, U. \& Morris, R. G. M. Synaptic tagging and long-term potentiation. Nature 385, 533-536 (1997).

16. Montague, P. R., Dayan, P., Person, C. \& Sejnowski, T. J. Bee foraging in uncertain environments using predictive hebbian learning. Nature 377, 725-728 (1995).

17. Lisman, J., Grace, A. A. \& Duzel, E. A neoHebbian framework for episodic memory; role of dopamine-dependent late LTP. Trends Neurosci. 34, 536-547 (2011).

18. Frémaux, N. \& Gerstner, W. Neuromodulated spike-timing-dependent plasticity, and theory of three-factor learning rules. Front. Neural Circuits 9, (2016).

19. Pennartz, C. A. M. The ascending neuromodulatory systems in learning by reinforcement: comparing computational conjectures with experimental findings. Brain Res. Rev. 21, 219-245 (1995).

20. Trabasso, T. \& Bower, G. H. Attention in Learning: Theory and Research. (Krieger Pub Co, 1968). 
931 21. Ahissar, M. \& Hochstein, S. Attentional control of early perceptual learning. Proc. Natl. Acad.

932

933

934

935

936

937

938

939

940

941

942

943

944

945

946

947

948

949

950

951

952

953

954

955

956

957

958

959

960

961

962

963

964

965

966

967

968

969

970

971

972

973

974

975

976

977

978

979

980

981

982

983

984

985

986

987 Sci. U.S.A. 90, 5718-5722 (1993).

22. Jiang, Y. \& Chun, M. M. Selective attention modulates implicit learning. Q.J.Exp.Psychol. 54, 1105-1124 (2001).

23. Vartak, D., Jeurissen, D., Self, M. W. \& Roelfsema, P. R. The influence of attention and reward on the learning of stimulus-response associations. Sci. Rep. 7, 9036 (2017).

24. Seitz, A. R., Kim, D. \& Watanabe, T. Rewards evoked learning of unconcsiously processed visual stimuli in adult humans. Neuron 61, 700-707 (2009).

25. Roelfsema, P. R., van Ooyen, A. \& Watanabe, T. Perceptual learning rules based on reinforcers and attention. Trends Cogn.Sci. 14, 64-71 (2010).

26. Jonikaitis, D. \& Deubel, H. Independent allocation of attention to eye and hand targets in coordinated eye-hand movements. Psychol. Sci. 22, 339-347 (2011).

27. Moore, T. Shape representations and visual guidance of saccadic eye movements. Science (80. ). 285, 1914-1917 (1999).

28. Roelfsema, P. R. \& van Ooyen, A. Attention-gated reinforcement learning of internal representations for classification. Neural Comp. 17, 2176-2214 (2005).

29. Lamme, V. A. F. \& Roelfsema, P. R. The distinct modes of vision offered by feedforward and recurrent processing. Trends Neurosci. 23, 571-579 (2000).

30. Moore, T. \& Armstrong, K. M. Selective gating of visual signals by microstimulation of frontal cortex. Nature 421, 370-373 (2003).

31. Lillicrap, T. P., Cownden, D., Tweed, D. B. \& Akerman, C. J. Random synaptic feedback weights support error backpropagation for deep learning. Nat. Commun. 7, 1-10 (2016).

32. Rombouts, J. O., Bohte, S. M. \& Roelfsema, P. R. How attention can create synaptic tags for the learning of working memories in sequential tasks. PloS Comput. Biol. 7, 1-34 (2015).

33. Mnih, V. et al. Human-level control through deep reinforcement learning. Nature 518, 529533 (2015).

34. Crick, F. The recent excitement about neural networks. Nature 337, 129-132 (1989).

35. Braitenberg, V. \& Schütz, A. Anatomy of the Cortex. (Springer-Verlag, 1991).

36. Mountcastle, V. B. in The Mindful Brain (eds. Edelman, G. M. \& Mountcastle, V. B.) (MIT Press, 1978).

37. Felleman, D. J. \& Van Essen, D. C. Distributed hierarchical processing in the primate cerebral cortex. Cereb.Cortex 1, 1-47 (1991).

38. Yamins, D. L. K. \& DiCarlo, J. J. Using goal-driven deep learning models to understand sensory cortex. Nat. Neurosci. 19, 356-365 (2016).

39. Sherman, S. M. Thalamus plays a central role in ongoing cortical functioning. Nat. Neurosci. 16, 533-41 (2016).

40. Callaway, E. M. Feedforward, feedback and inhibitory connections in primate visual cortex. Neural Networks 17, 625-632 (2004).

41. Harris, K. D. \& Mrsic-Flogel, T. D. Cortical connectivity and sensory coding. Nature 503, 51-8 (2013).

42. Douglas, R. \& Martin, K. A. C. Neuronal circuits of the neocortex. Annual Reviews in Neuroscience 27, 419-451 (2004).

43. Markov, N. T. et al. Cortical high-density counterstream architectures. Science (80-. ). 342, 578- (2013).

44. Ullman, S. Sequence seeking and counterstreams: a computational model for bidirectional information flow in the visual cortex. Cereb.Cortex 5, 1-11 (1995).

45. Harris, K. D. \& Shepherd, G. M. G. The neocortical circuit: themes and variations. Nat. Neurosci. 18, 170-181 (2015).

46. Feldmeyer, D. Excitatory neuronal connectivity in the barrel cortex. Front. Neuroanat. 6, 24 (2012).

47. Maunsell, J. H. R. \& Gibson, J. R. Visual response latencies in striate cortex of the macaque monkey. J. Neurophysiol. 68, 1332-1344 (1992).

48. Nowak, L. G., Munk, M. H. J., Girard, P. \& Bullier, J. Visual latencies in areas V1 and V2 of the macaque monkey. Visual Neuroscience 12, 371-384 (1995).

49. van Kerkoerle, T., Self, M. W. \& Roelfsema, P. R. Effects of attention and working memory in the different layers of monkey primary visual cortex. Nat. Commun. 8, 1-12 (2017).

50. Self, M. W., van Kerkoerle, T., Supèr, H. \& Roelfsema, P. R. Distinct roles of the cortical layers 
988

989

990

991

992

993

994

995

996

997

998

999

1000

1001

1002

1003

1004

1005

1006

1007

1008

1009

1010

1011

1012

1013

1014

1015

1016

1017

1018

1019

1020

1021

1022

1023

1024

1025

1026

1027

1028

1029

1030

1031

1032

1033

1034

1035

1036

1037

1038

1039

1040

1041

1042

1043

1044 of area V1 in figure-ground segregation. Curr.Biol. 23, 2121-2129 (2013).

51. Constantinople, C. M. \& Bruno, R. M. Deep cortical layers are activated by thalamus. Science (80-. ). 340, 1591-1594 (2013).

52. Morgenstern, N. A., Bourg, J. \& Petreanu, L. Multilaminar networks of cortical neurons integrate common inputs from sensory thalamus. Nat. Neurosci. 19, 1034-1040 (2016).

53. Bolz, J. \& Gilbert, C. D. Generation of end-inhibition in the visual cortex via interlaminar connections. Nature 320, 362-365 (1986).

54. Olsen, S. R., Bortone, D. S., Adesnik, H. \& Scanziani, M. Gain control by layer six in cortical circuits of vision. Nature 483, 47-52 (2012).

55. Bortone, D. S., Olsen, S. R. \& Scanziani, M. Translaminar inhibitory cells recruited by layer 6 corticothalamic neurons suppress visual cortex. Neuron 82, 474-485 (2014).

56. Rockland, K. S. \& Virga, A. Terminal arbors of individual 'feedback' axons projecting from area V2 to V1 in the macaque monkey: a study using immunohistochochemistry of anterogradely transported phaseolus vulgaris-leucoagglutinin. J.Comp. Neurol. 285, 54-72 (1989).

57. Larkum, M. E. A cellular mechanism for cortical associations: an organizing principle for the cerebral cortex. Trends Neurosci. 36, 141-149 (2013).

58. Schneider, D. M., Nelson, A. \& Mooney, R. A synaptic and circuit basis for corollary discharge in the auditory cortex. Nature 513, 189-194 (2014).

59. Lee, S., Kruglikov, I., Huang, Z. J., Fishell, G. \& Rudy, B. A disinhibitory circuit mediates motor integration in the somatosensory cortex. Nat. Neurosci. 16, 1662-1670 (2013).

60. van Versendaal, D. \& Levelt, C. N. Inhibitory interneurons in visual cortical plasticity. Cell. Mol. Life Sci. 73, 3677-3691 (2016).

61. Deschênes, M., Veinante, P. \& Zhang, Z. W. The organization of corticothalamic projections: reciprocity versus parity. Brain Res. Rev. 28, 286-308 (1998).

62. Veinante, P., Lavallée, P. \& Deschênes, M. Corticothalamic projections from layer 5 of the vibrissal barrel cortex in the rat. J. Comp. Neurol. 424, 197-204 (2000).

63. Jones, E. G. Thalamus. (Cambridge University Press, 2007).

64. Roth, M. M. et al. Thalamic nuclei convey diverse contextual information to layer 1 of visual cortex. Nat. Neurosci. 19, 299-307 (2016).

65. Meyer, H. S. et al. Cell type-specific thalamic innervation in a column of rat vibrissal cortex. Cereb. Cortex 20, 2287-2303 (2010).

66. Ohno, S. et al. A morphological analysis of thalamocortical axon fibers of rat posterior thalamic nuclei: A single neuron tracing study with viral vectors. Cereb. Cortex 22, 2840-2857 (2012).

67. Lu, S. M. \& Lin, R. C. Thalamic afferents of the rat barrel cortex: a light- and electronmicroscopic study using Phaseolus vulgaris leucoagglutinin as an anterograde tracer. Somatosens. Mot. Res. 10, 1-16 (1993).

68. Mease, R. A., Metz, M. \& Groh, A. Cortical sensory responses are enhanced by the higherorder thalamus. Cell Rep. 14, 208-215 (2016).

69. Groh, A. et al. Convergence of cortical and sensory driver inputs on single thalamocortical cells. Cereb. Cortex 24, 3167-3179 (2014).

70. Ahissar, E., Sosnik, R. \& Haidarliu, S. Transformation from temporal to rate coding in a somatosensory thalamocortical pathway. Nature 406, 302-306 (2000).

71. Moore, J. D., Mercer Lindsay, N., Deschênes, M. \& Kleinfeld, D. Vibrissa Self-Motion and Touch Are Reliably Encoded along the Same Somatosensory Pathway from Brainstem through Thalamus. PLoS Biol. 13, 1-28 (2015).

72. Guo, Z. V. et al. Maintenance of persistent activity in a frontal thalamocortical loop. Nature 545, 181-186 (2017).

73. Kwon, S. E., Yang, H., Minamisawa, G. \& O'Connor, D. H. Sensory and decision-related activity propagate in a cortical feedback loop during touch perception. Nat. Neurosci. 19, (2016).

74. Self, M., Kooijmans, R. N., Supèr, H., Lamme, V. A. F. \& Roelfsema, P. R. Different glutamate receptors convey feedforward and recurrent processing in macaque V1. Proc. Natl. Acad. Sci. U.S.A. 109, 11031-11036 (2012).

75. Daw, N. W., Stein, P. S. G. \& Fox, K. The role of NMDA receptors in information processing. Annu. Rev. Neurosci. 16, 207-222 (1993).

76. Rivadulla, C., Martinez, L. M., Varela, C. \& Cudeiro, J. Completing the corticofugal loop: A visual role for the corticogeniculate type 1 metabotropic glutamate receptor. J. Neurosci. 22, 
1045

1046

1047

1048

1049

1050

1051

1052

1053

1054

1055

1056

1057

1058

1059

1060

1061

1062

1063

1064

1065

1066

1067

1068

1069

1070

1071

1072

1073

1074

1075

1076

1077

1078

1079

1080

1081

1082

1083

1084

1085

1086

1087

1088

1089

1090

1091

1092

1093

1094

1095

1096

1097

1098

1099

1100

1101

2956-2962 (2002).

77. Gambino, F. et al. Sensory-evoked LTP driven by dendritic plateau potentials in vivo. Nature 515, 116-119 (2014).

78. Klink, P. C., Dagnino, B., Gariel-Mathis, M. A. \& Roelfsema, P. R. Distinct feedforward and feedback effects of microstimulation in visual cortex reveal neural mechanisms of texture segregation. Neuron 95, 209-220 (2017).

79. Tritsch, N. X. \& Sabatini, B. L. Dopaminergic modulation of synaptic transmission in cortex and striatum. Neuron 76, 33-50 (2012).

80. Picciotto, M. R., Higley, M. J. \& Mineur, Y. S. Acetylcholine as a neuromodulator: cholinergic signaling shapes nervous system function and behavior. Neuron 76, 116-129 (2012).

81. Eggermann, E., Kremer, Y., Crochet, S. \& Petersen, C. C. H. Cholinergic signals in mouse barrel cortex during active whisker sensing. Cell Rep. 1-7 (2014). doi:10.1016/j.celrep.2014.11.005

82. Gu, Q. Neuromodulatory transmitter system in the cortex and their role in cortical plasticity. Neuroscience 111, 814-835 (2002).

83. Lesch, K. P. \& Waider, J. Serotonin in the modulation of neural plasticity and networks: implications for neurodevelopmental disorders. Neuron 76, 175-191 (2012).

84. Froemke, R. C. Plasticity of Cortical Excitatory-Inhibitory Balance. Annu. Rev. Neurosci. 38, 195-219 (2015).

85. Hayashi-takagi, A. et al. Labelling and optical erasure of synaptic memory traces in the motor cortex. Nature 525, 333-338 (2015).

86. Xiong, Q., Znamenskiy, P. \& Zador, A. M. Selective corticostriatal plasticity during acquisition of an auditory discrimination task. Nature 521, 348-351 (2015).

87. Jin, X. \& Costa, R. M. Start / stop signals emerge in nigrostriatal circuits during sequence learning. Nature 466, 457-462 (2010).

88. Liu, B., Huberman, A. D. \& Scanziani, M. Cortico-fugal output from visual cortex promotes plasticity of innate motor behaviour. Nature 538, 383-387 (2016).

89. Schoups, A., Vogels, R., Qian, N. \& Orban, G. A. Practising orientation identification improves orientation coding in V1 neurons. Nature 412, 549-553 (2001).

90. Poort, J. et al. Learning enhances sensory and multiple non-sensory representations in primary visual cortex. Neuron 86, 1478-1490 (2015).

91. Goltstein, P. M., Coffey, E. B. J., Roelfsema, P. R. \& Pennartz, C. M. a. In vivo two-photon Ca2+ imaging reveals selective reward effects on stimulus-specific assemblies in mouse visual cortex. J. Neurosci. 33, 11540-11555 (2013).

92. Freedman, D. J. \& Assad, J. A. Experience dependent representation of visual categories in parietal cortex. Nature 443, 85-88 (2006).

93. Makino, H. \& Komiyama, T. Learning enhances the relative impact of top-down processing in the visual cortex. Nat. Neurosci. 18, (2015).

94. Bajo, V. M., Nodal, F. R., Moore, D. R. \& King, A. J. The descending corticocollicular pathway mediates learning-induced auditory plasticity. Nat. Neurosci. 13, 253-260 (2010).

95. Brosch, T., Neumann, H. \& Roelfsema, P. R. Reinforcement Learning of Linking and Tracing Contours in Recurrent Neural Networks. PLoS Comput. Biol. 11, 1-36 (2015).

96. Pooresmaeili, A., Poort, J. \& Roelfsema, P. R. Simultaneous selection by object-based attention in visual and frontal cortex. Proc. Natl. Acad. Sci. U. S. A. 111, 6467-72 (2014).

97. Self, M. W. et al. The effects of context and attention on spiking activity in human early visual cortex. PLoS Biol. 14, 1-26 (2016).

98. Houtkamp, R., Spekreijse, H. \& Roelfsema, P. R. A gradual spread of attention during mental curve tracing. Percept. Psychophys. 65, 1136-1144 (2003).

99. Roelfsema, P. R. \& Spekreijse, H. The representation of erroneously perceived stimuli in the primary visual cortex. Neuron 31, 853-863 (2001).

100. Khayat, P. S., Pooresmaeili, A. \& Roelfsema, P. R. Time course of attentional modulation in the frontal eye field during curve tracing. J. Neurophysiol. 101, (2009).

101. Roelfsema, P. R. Cortical algorithms for perceptual grouping. Annu. Rev. Neurosci. 29, 203227 (2006).

102. Reynolds, J. H. \& Chelazzi, L. Attentional modulation of visual processing. Annu. Rev. Neurosci. 27, 611-647 (2004).

103. Zhou, H., Schafer, R. J. \& Desimone, R. Pulvinar-cortex interactions in vision and attention. Neuron 89, 209-220 (2016). 
1102

1103

1104

1105

1106

1107

1108

1109

1110

1111

1112

1113

1114

1115

1116

1117

1118

1119

1120

1121

1122

1123

1124

1125

1126

1127

1128

1129

1130

1131

1132

1133

1134

1135

1136

1137

1138

1139

1140

1141

1142

1143

1144

1145

1146

1147

1148

1149

1150

1151

1152

1153

1154

1155

1156

1157

1158

104. Purushothaman, G., Marion, R., Li, K. \& Casagrande, V. A. Gating and control of primary visual cortex by pulvinar. Nat. Neurosci. 15, 905-912 (2012).

105. Robinson, D. L. \& Petersen, S. E. The pulvinar and visual salience. Trends Neurosci. 15, 127132 (1992).

106. Chalupa, L. M., Coyle, R. S. \& Lindsley, D. B. Effect of pulvinar lesions on visual pattern discrimination in monkeys. J. Neurophysiol. 39, 354-369 (1976).

107. Manita, S. et al. A top-down cortical circuit for accurate sensory perception. Neuron 86, 13041316 (2015).

108. Xu, N. et al. Nonlinear dendritic integration of sensory and motor input during an active sensing task. Nature 492, 247-51 (2012).

109. Sachidhanandam, S., Sreenivasan, V., Kyriakatos, A., Kremer, Y. \& Petersen, C. C. H. Membrane potential correlates of sensory perception in mouse barrel cortex. Nat. Neurosci. 16, 1671-7 (2013).

110. Takahashi, N., Oertner, T. G., Hegemann, P. \& Larkum, M. E. Active cortical dendrites modulate perception. Science (80-. ). 354, 1587-1590 (2016).

111. Gambino, F. \& Holtmaat, A. Spike-timing-dependent potentiation of sensory surround in the somatosensory cortex is facilitated by deprivation-mediated disinhibition. Neuron 75, 490502 (2012).

112. Letzkus, J. J. et al. A disinhibitory microcircuit for associative learning in the auditory cortex. Nature 480, 331-335 (2011).

113. Letzkus, J. J., Wolff, S. B. E. \& Lüthi, A. Disinhibition, a Circuit Mechanism for Associative Learning and Memory. Neuron 88, 264-276 (2015).

114. Lee, S., Hjerling-Leffler, J., Zagha, E., Fishell, G. \& Rudy, B. The largest group of superficial neocortical GABAergic interneurons expresses ionotropic serotonin receptors. J. Neurosci. 30, 16796-16808 (2010).

115. Wall, N. R. et al. Brain-wide maps of synaptic input to cortical interneurons. J. Neurosci. 36, 4000-4009 (2016).

116. Audette, N. J., Urban-Ciecko, J., Matsushita, M. \& Barth, A. L. POm thalamocortical input drives layer-specific microcircuits in somatosensory cortex. Cereb. Cortex 1-17 (2017). doi:10.1093/cercor/bhx044

117. Pi, H.-J. et al. Cortical interneurons that specialize in disinhibitory control. Nature 503, 521524 (2013).

118. Fu, Y. et al. A cortical circuit for gain control by behavioral state. Cell 156, 1139-52 (2014).

119. Pfeffer, C. K., Xue, M., He, M., Huang, Z. J. \& Scanziani, M. Inhibition of inhibition in visual cortex: the logic of connections between molecularly distinct interneurons. Nat. Neurosci. 16, 1068-1076 (2013).

120. Wang, Y. et al. Anatomical, physiological and molecular properties of Martinotti cells in the somatosensory cortex of the juvenile rat. J. Physiol. 561, 65-90 (2004).

121. van Versendaal, D. et al. Elimination of inhibitory synapses Is a major component of adult ocular dominance plasticity. Neuron 74, 374-383 (2012).

122. Kubota, Y., Hatada, S., Kondo, S., Karube, F. \& Kawaguchi, Y. Neocortical inhibitory terminals innervate dendritic spines targeted by thalamocortical afferents. J. Neurosci. 27, 1139-1150 (2007).

123. Palmer, L., Murayama, M. \& Larkum, M. Inhibitory regulation of dendritic activity in vivo. Front. Neural Circuits 6, 1-10 (2012).

124. Chen, S. X., Kim, A. N., Peters, A. J. \& Komiyama, T. Subtype-specific plasticity of inhibitory circuits in motor cortex during motor learning. Nat. Neurosci. 18, 1109-1115 (2015).

125. Cichon, J. \& Gan, W. Branch-specific dendritic Ca2+ spikes cause persistent synaptic plasticity. Nature 520, 180-185 (2015).

126. Fu, Y., Kaneko, M. K., Tang, Y., Alvarez-Buylla, A. \& Stryker, M. P. A cortical disinhibitory circuit for enhancing adult plasticity. Elife 4, :e05558 (2015).

127. Higley, M. J. Localized GABAergic inhibition of dendritic Ca2+ signalling. Nat. Rev. Neurosci. 15, 567-572 (2014).

128. Bittner, K. C., Milstein, A. D., Grienberger, C., Romani, S. \& Magee, J. C. Behavioral time scale synaptic plasticity underlies CA1 place fields. Science (80-. ). 357, 1033-1036 (2017).

129. Basu, J. et al. Gating of hippocampal activity, plasticity, and memory by entorhinal cortex long-range inhibition. Science (80-. ). 351, aaa5694-aaa5694 (2016). 
1159 130. Smith, S. L., Smith, I. T., Branco, T. \& Häusser, M. Dendritic spikes enhance stimulus selectivity in cortical neurons in vivo. Nature 503, 115-20 (2013).

1161

131. Dahmen, J. C., Hartley, D. E. H. \& King, A. J. Stimulus-Timing-Dependent Plasticity of Cortical Frequency Representation. J. Neurosci. 28, 13629-13639 (2008).

132. Pawlak, V. \& Kerr, J. N. D. Dopamine receptor activation is required for corticostriatal spiketiming-dependent plasticity. J Neurosci 28, 2435-2446 (2008).

1165

1166

1167

1168

1169

1170

1171

1172

1173

1174

1175

1176

1177

1178

1179

1180

1181

1182

1183

1184

1185

1186

1187

1188

1189

1190

1191

1192

1193

1194

1195

1196

1197

1198

1199

1200

1201

1202

1203

1204

1205

1206

1207

1208

1209

1210

1211

1212

1213

1214

1215
133. Pawlak, V., Greenberg, D. S., Sprekeler, H., Gerstner, W. \& Kerr, J. N. D. Changing the responses of cortical neurons from sub- To suprathreshold using single spikes in vivo. Elife 2013, 1-18 (2013).

134. Haas, H. L., Sergeeva, O. A. \& Selbach, O. Histamine in the central nervous system. Physiol.Rev. 88, 1183-1241 (2008).

135. Montague, P. R., Hyman, S. E. \& Cohen, J. D. Computational roles for dopamine in behavioral control. Nature 760-767 (2004).

136. Schultz, W. Multiple dopamine functions at different time courses. Annu. Rev. Neurosci. 30, 259-288 (2007).

137. Bromberg-Martin, E. S., Matsumoto, M. \& Hikosaka, O. Dopamine in motivational control: rewarding, aversive, and alerting. Neuron 68, 815-834 (2010).

138. Bao, S., Chan, V. T. \& Merzenich, M. M. Cortical remodelling induced by activity of ventral tegmental dopamine neurons. Nature 412, 79-81 (2001).

139. Lammel, S. et al. Input-specific control of reward and aversion in the ventral tegmental area. Nature 491, 212-217 (2012).

140. Zaborszky, L. et al. Neurons in the basal forebrain project to the cortex in a complex topographic organization that reflects corticocortical connectivity patterns: an experimental study based on retrograde tracing and 3D reconstruction. Cereb.Cortex 25, 118-137 (2015).

141. Kim, J.-H. et al. Selectivity of neuromodulatory projections from the basal forebrain and locus ceruleus to primary sensory cortices. J. Neurosci. 36, 5314-5327 (2016).

142. Kawai, H., Lazar, R. \& Metherate, R. Nicotinic control of axon excitability regulates thalamocortical transmission. Nat. Neurosci. 10, 1168-1175 (2007).

143. Férézou, I. et al. 5-HT3 receptors mediate serotonergic fast synaptic excitation of neocortical vasoactive intestinal peptide/cholecystokinin interneurons. J. Neurosci. 22, 7389-7397 (2002).

144. Pinto, L. et al. Fast modulation of visual perception by basal forebrain cholinergic neurons. Nat. Neurosci. 16, 1857-1863 (2013).

145. Hangya, B., Ranade, S. P., Lorenc, M. \& Kepecs, A. Central cholinergic neurons are rapidly recruited by reinforcement feedback. Cell 162, 1155-1168 (2015).

146. Richardson, R. T. \& DeLong, M. R. Nucleus basalis of Meynert neuronal activity during a delayed response task in monkey. Brain Res. 399, 364-368 (1986).

147. Chubykin, A. A., Roach, E. B., Bear, M. F. \& Shuler, M. G. H. A cholinergic mechanism for reward timing within primary visual cortex. Neuron 77, 723-735 (2013).

148. Kilgard, M. P. \& Merzenich, M. M. Cortical map reorganization enabled by nucleus basalis activity. Science (80-. ). 279, 1714-1718 (1998).

149. Froemke, R. C. et al. Long-term modification of cortical synapses improves sensory perception. Nat Neurosci 16, 79-88 (2013).

150. Bakin, J. S. \& Weinberger, N. M. Induction of a phsyiological memory in the cerebral cortex by stimulation of the nucleus basalis. Proc. Natl. Acad. Sci. U.S.A. 93, 11219-11224 (1996).

151. Froemke, R. C., Merzenich, M. M. \& Schreiner, C. E. A synaptic memory trace for cortical receptive field plasticity. Nature 450, 425-429 (2007).

152. Juliano, S. L., Ma, W. \& Eslin, D. Cholinergic depletion prevents expansion of topographic maps in somatosensory cortex. Proc. Natl. Acad. Sci. U.S.A. 88, 780-784 (1991).

153. Warburton, E. C. et al. Cholinergic neurotransmission is essential for perirhinal cortical plasticity and recognition memory. Neuron 38, 987-996 (2003).

154. Easton, A., Ridley, R. M., Baker, H. F. \& Gaffan, D. Unilateral lesions of the cholinergic basal forebrain and fornix in one hemisphere and inferior temporal cortex in the opposite hemisphere produce severe learning impairements in rhesus monkeys. Cereb.Cortex 12, 729736 (2002).

155. Winkler, J., Suhr, S. T., Gage, F. H., Thal, L. J. \& Fisher, L. J. Essential role of neocortical acetylcholine in spatial memory. Nature 375, 484-487 (1995).

156. Jacobs, B. L. \& Azmitia, E. C. Structure and function of the brain serotonin system. Physiol Rev 
1216

1217

1218

1219

1220

1221

1222

1223

1224

1225

1226

1227

1228

1229

1230

1231

1232

1233

1234

1235

1236

1237

1238

1239

1240

1241

1242

1243

1244

1245

1246

1247

1248

1249

1250

1251

1252

1253

1254

1255

1256

1257

1258

1259

1260

1261

1262

1263

1264

1265

1266

1267

1268

1269

1270

1271

1272
72, 165-229 (1992).

157. Celada, P., Puig, M. V. \& Artigas, F. Serotonin modulation of cortical neurons and networks. Front. Integr. Neurosci. 7, 25 (2013).

158. Nakamura, K., Matsumoto, M. \& Hikosaka, O. Reward-dependent modulation of neuronal activity in the primate dorsal raphe nucleus. J. Neurosci. 28, 5331-5343 (2008).

159. Ranade, S. P. \& Mainen, Z. F. Transient firing of dorsal raphe neurons encodes diverse and specific sensory, motor, and reward events. J. Neurophysiol. 102, 3026-37 (2009).

160. Cohen, J. Y., Amoroso, M. W. \& Uchida, N. Serotonergic neurons signal reward and punishment on multiple timescales. Elife 4, 1-25 (2015).

161. Bromberg-martin, E. S., Hikosaka, O. \& Nakamura, K. Coding of task reward value in the dorsal raphe nucleus. J.Neurosci. 30, 6262-6272 (2010).

162. Liu, Z. et al. Dorsal raphe neurons signal reward through 5-HT and glutamate. Neuron 81, 1360-1374 (2014).

163. Nakamura, K. The role of the dorsal raphé nucleus in reward-seeking behavior. Front. Integr. Neurosci. 7, 60 (2013).

164. Jitsuki, S. et al. Serotonin mediates cross-modal reorganization of cortical circuits. Neuron 69, 780-792 (2011).

165. Sara, S. J. \& Bouret, S. Orienting and reorienting: the locus coeruleus mediates cognition through arousal. Neuron 76, 130-141 (2012).

166. Bouret, S. \& Richmond, B. J. Sensitivity of locus ceruleus neurons to reward value for goaldirected actions. J. Neurosci. 35, 4005-4014 (2015).

167. Martins, A. R. O. \& Froemke, R. C. Coordinated forms of noradrenergic plasticity in the locus coeruleus and primary auditory cortex. Nat. Neurosci. 18, 1-12 (2015).

168. Devilbiss, D. M. et al. Phasic and tonic patterns of locus coeruleus output differentially modulate sensory network function in the awake rat phasic and tonic patterns of locus coeruleus output Differentially Modulate Sensory Network Function in the Awake Rat. J.Neurophysiol. 105, 69-87 (2011).

169. Pawlak, V., Wickens, J. R., Kirkwood, A. \& Kerr, J. N. D. Timing is not everything: Neuromodulation opens the STDP gate. Front. Synaptic Neurosci. 2, 1-14 (2010).

170. Hu, H. et al. Emotion enhances learning via norepinephrine regulation of AMPA-receptor trafficking. Cell 131, 160-173 (2007).

171. Johansen, J. P. et al. Hebbian and neuromodulatory mechanisms interact to trigger associative memory formation. Proc. Natl. Acad. Sci. 201421304 (2014). doi:10.1073/pnas.1421304111

172. Urbanczik, R. \& Senn, W. Reinforcement learning in populations of spiking neurons. Nat. Neurosci. 12, 250-252 (2009).

173. Seol, G. H. et al. Neuromodulators control the polarity of spike-timing-dependent plasticity. Neuron 55, 919-929 (2007).

174. He, K. et al. Distinct eligibility traces for LTP and LTD in cortical synapses. Neuron $\mathbf{8 8 , 5 2 8 - 5 3 8}$ (2015).

175. Cassenaer, S. \& Laurent, G. Conditional modulation of spike-timing-dependent plasticity for olfactory learning. Nature 482, 47-52 (2012).

176. Redondo, R. L. \& Morris, R. G. M. Making memories last: the synaptic tagging and capture hypothesis. Nat. Rev. Neurosci. 12, 17-30 (2011).

177. Clopath, C., Ziegler, L., Vasilaki, E., Büsing, L. \& Gerstner, W. Tag-trigger-consolidation: A model of early and late long-term-potentiation and depression. PLoS Comput. Biol. 4, (2008).

178. Nadim, F. \& Bucher, D. Neuromodulation of neurons and synapses. Curr. Opin. Neurobiol. 29, 48-56 (2014).

179. Blundon, J. A., Bayazitov, I. T. \& Zakharenko, S. S. Presynaptic gating of postsynaptically expressed plasticity at mature thalamocortical synapses. J.Neurosci. 31, 16012-25 (2011).

180. Brzosko, Z., Schultz, W. \& Paulsen, O. Retroactive modulation of spike timingdependent plasticity by dopamine. Elife 4, 1-13 (2015).

181. Güçlü, U. \& van Gerven, M. A. J. Deep neural networks reveal a gradient in the complexity of neural representations across the ventral stream. J. Neurosci. 35, 10005-10014 (2015).

182. Urbanczik, R. \& Senn, W. Learning by the dendritic prediction of somatic spiking. Neuron $\mathbf{8 1}$ 521-528 (2014).

183. Schiess, M., Urbanczik, R. \& Senn, W. Somato-dendritic Synaptic Plasticity and Errorbackpropagation in Active Dendrites. PLoS Comput. Biol. 12, 1-18 (2016). 
1273

1274

1275

1276

1277

1278

1279

1280

1281

1282

1283

1284

1285

1286

1287

1288
184. Scellier, B. \& Bengio, Y. Equilibrium Propagation: Bridging the Gap Between Energy-Based Models and Backpropagation. arXiv 1-21 (2017). doi:10.3389/fncom.2017.00024

185. Marblestone, A., Wayne, G. \& Kording, K. Towards an integration of deep learning and neuroscience. Front.Comput. Neurosci. 10, 1-41 (2016).

186. Laramée, M.-E. \& Boire, D. Visual cortical areas of the mouse: comparison of parcellation and network structure with primates. Front. Neural Circuits 8, 1-16 (2015).

187. Berezovskii, V. K., Nassi, J. J. \& Born, R. T. Segregation of feedforward and feedback projections in mouse visual cortex. J.Comp. Neurol. 519, 3672-3683 (2011).

188. Agnati, L. F., Guidolin, D., Guescini, M., Genedani, S. \& Fuxe, K. Understanding wiring and volume transmission. Brain Res. Rev. 64, 137-159 (2010).

189. Knott, G. W., Quairiaux, C., Genoud, C. \& Welker, E. Formation of dendritic spines with GABAergic synapses by whisker stimulation in induced adult mice. Neuron 34, 265-273 (2002). 
Fig. 1

Feedforward

[Sensory processing]

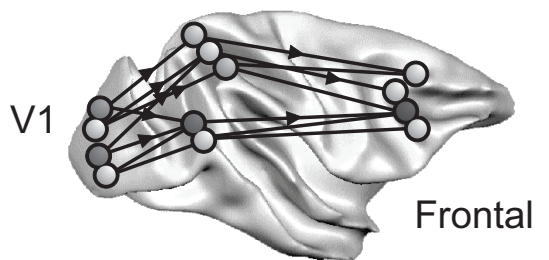

\section{Feedback}

[Motor selection and attentional tagging]

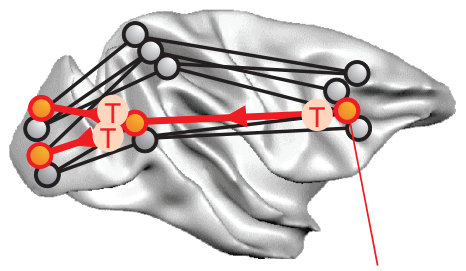

Selected motor program

T Synaptic tag

\section{Neuromodulator}

[Reward/ punishment]

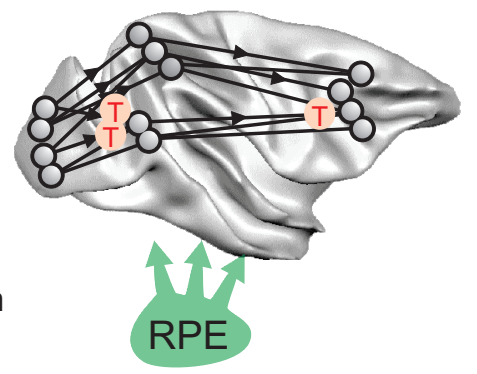

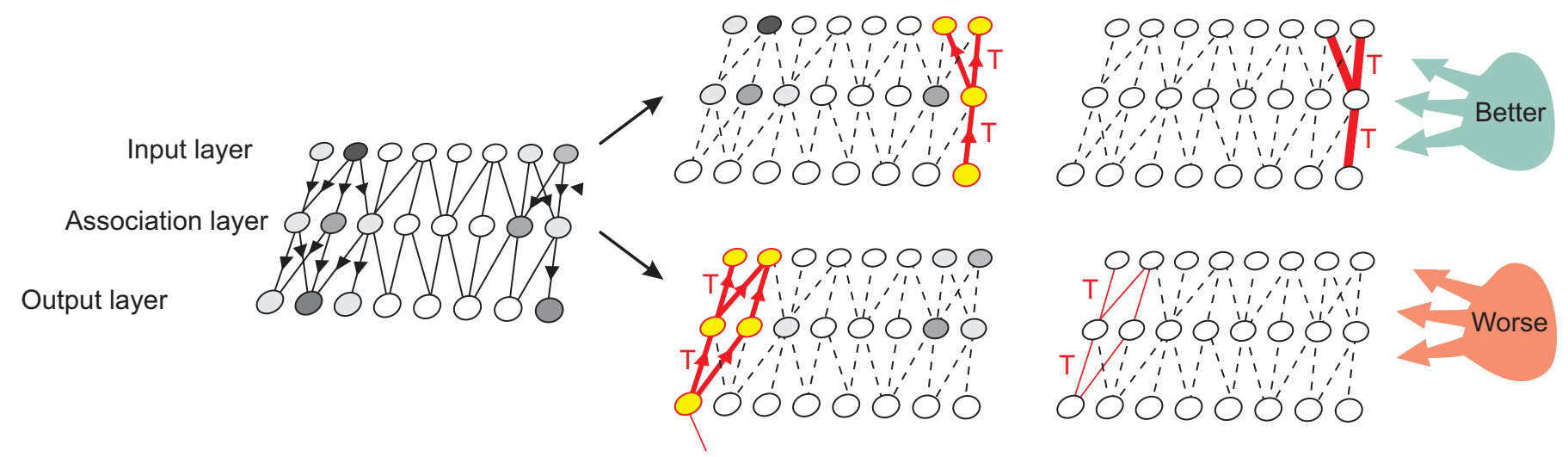

Selected motor program

Time 
Fig. 2

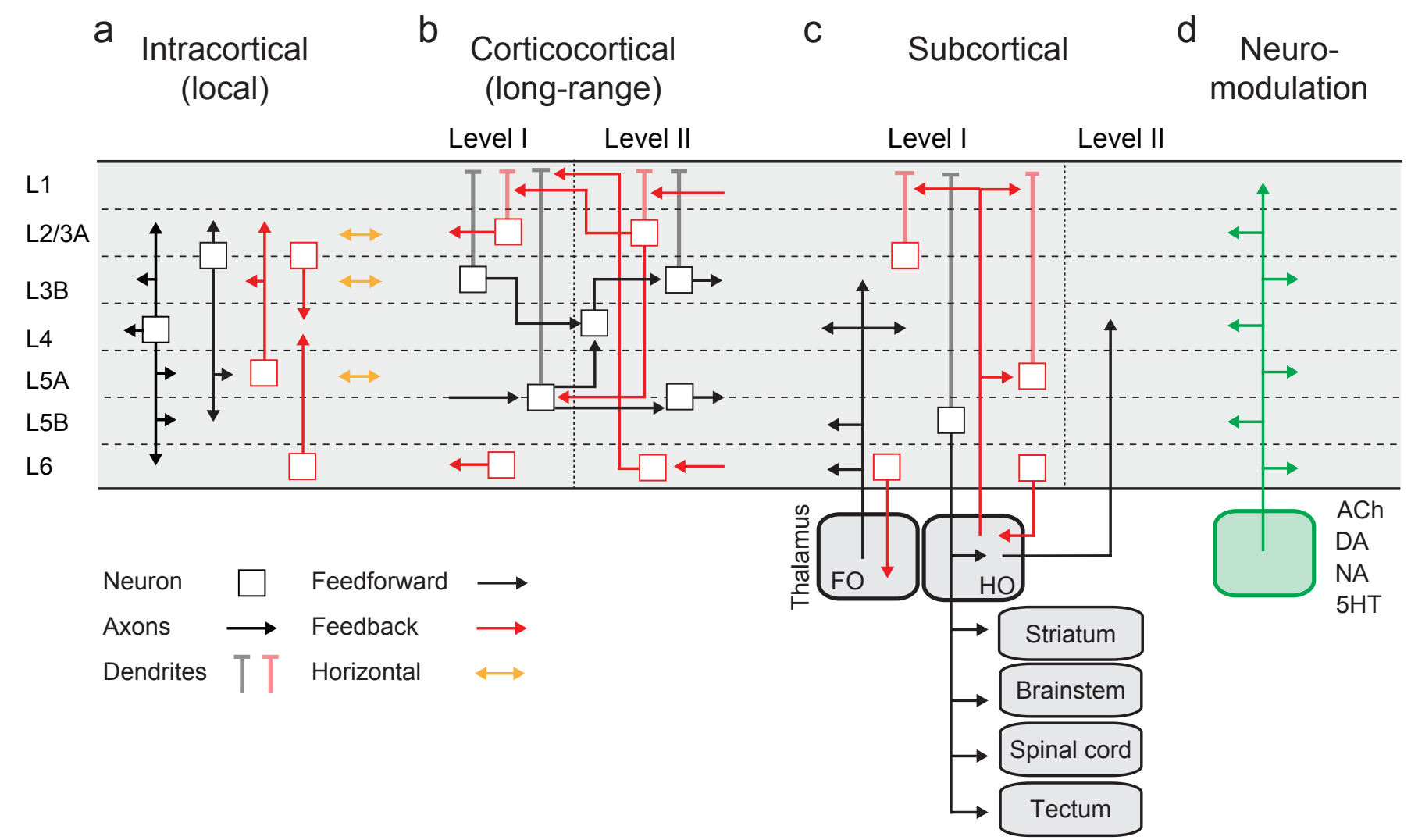


Fig. 3

a
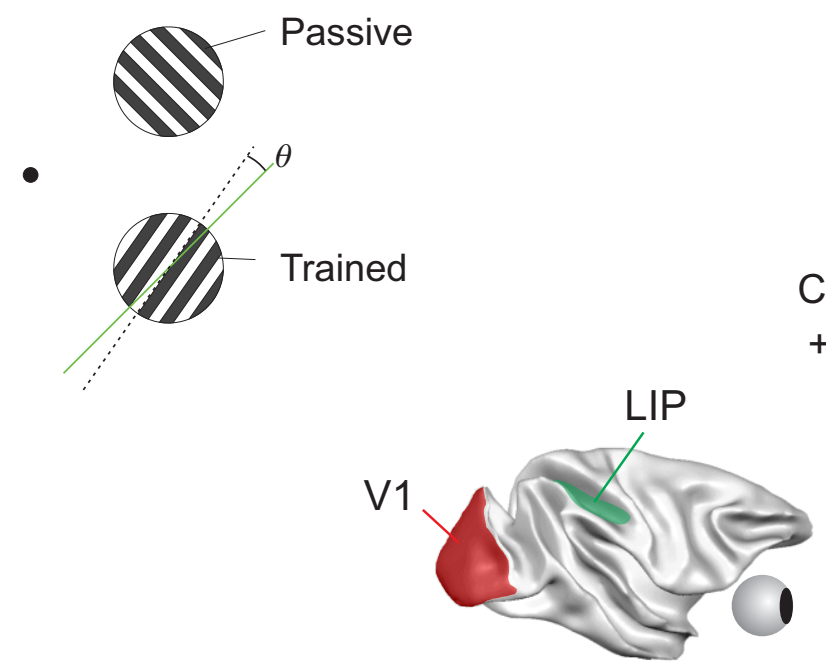

Area V1

b

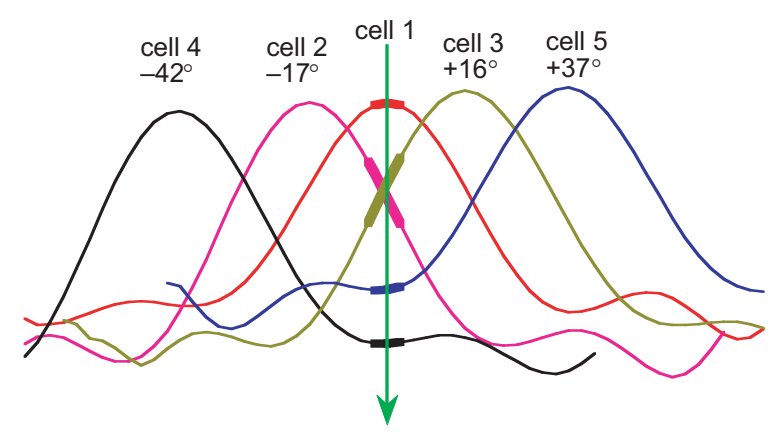

C

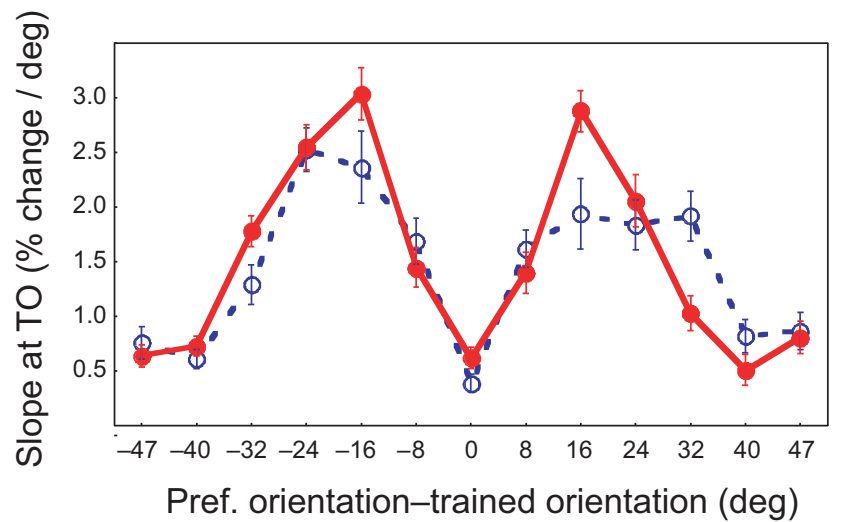

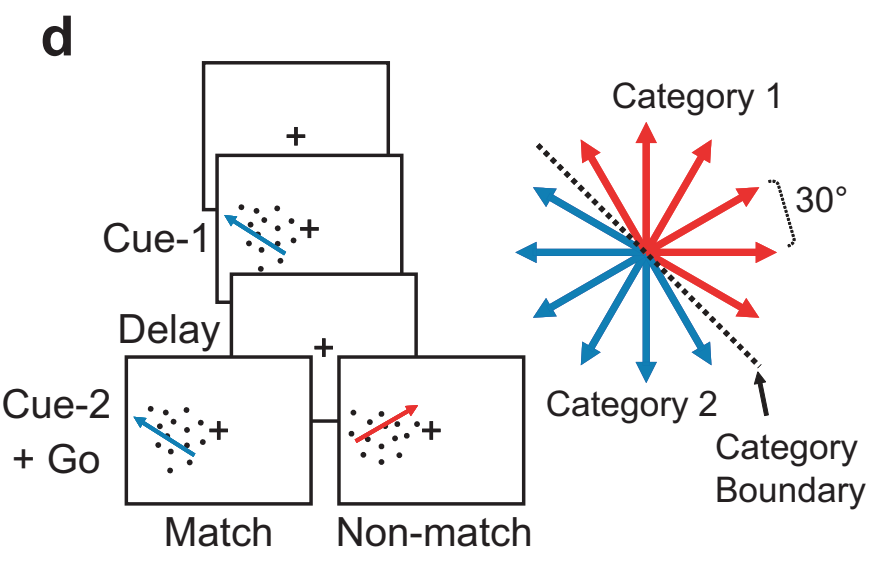

Area LIP

e
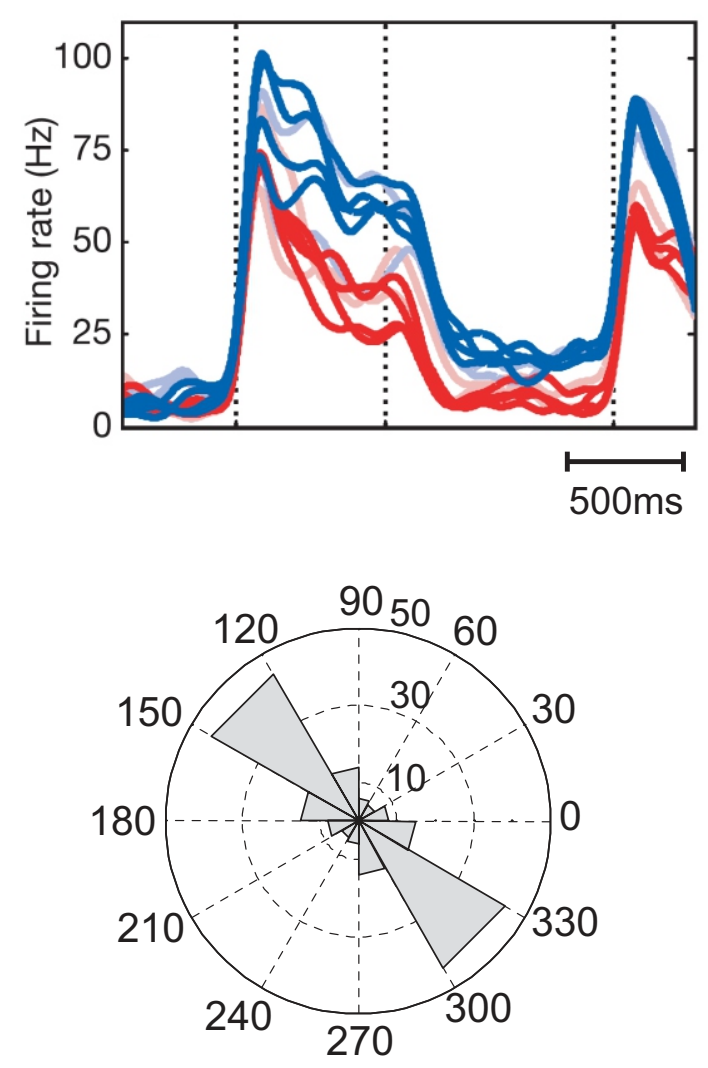
a

Fixation

Stimulus

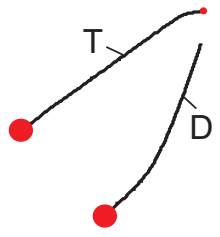

b

Target ( $\mathrm{T}$ )

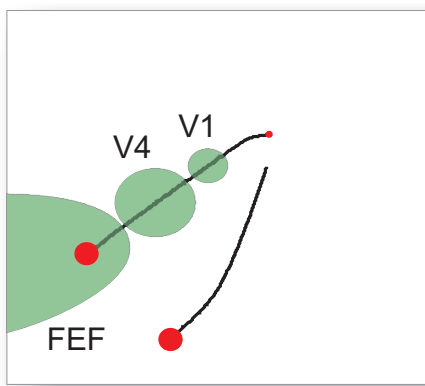

C

Area V1

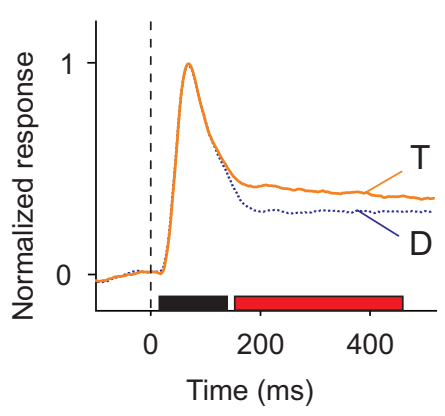

Area V4

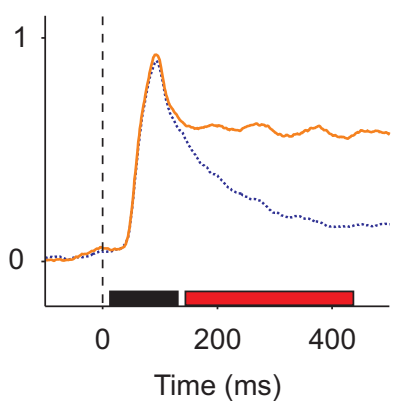

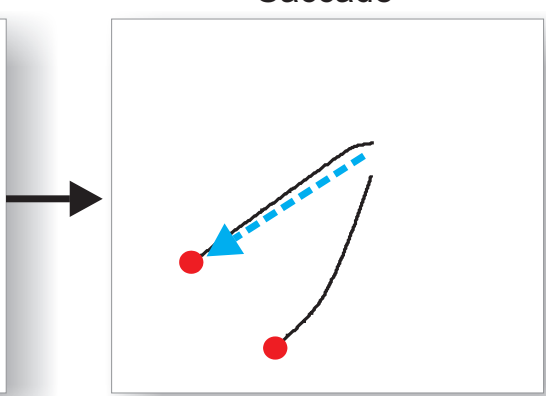

Saccade

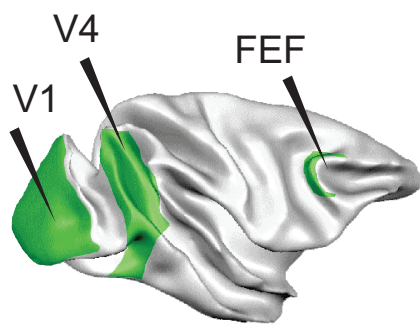

Area FEF

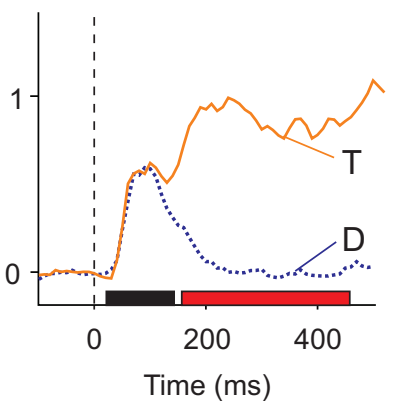

feedforward
recurrent

Fig. 4 
Fig. 5

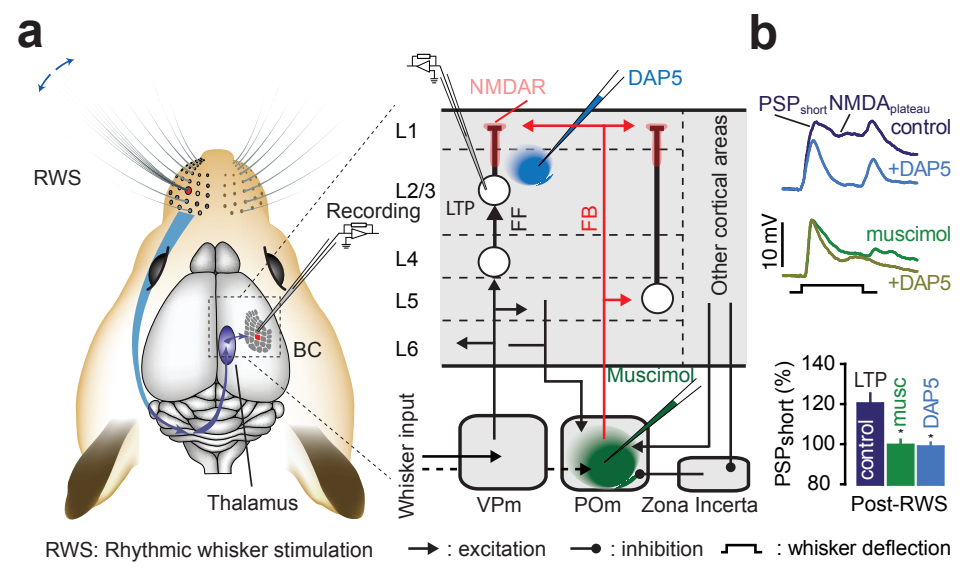

C

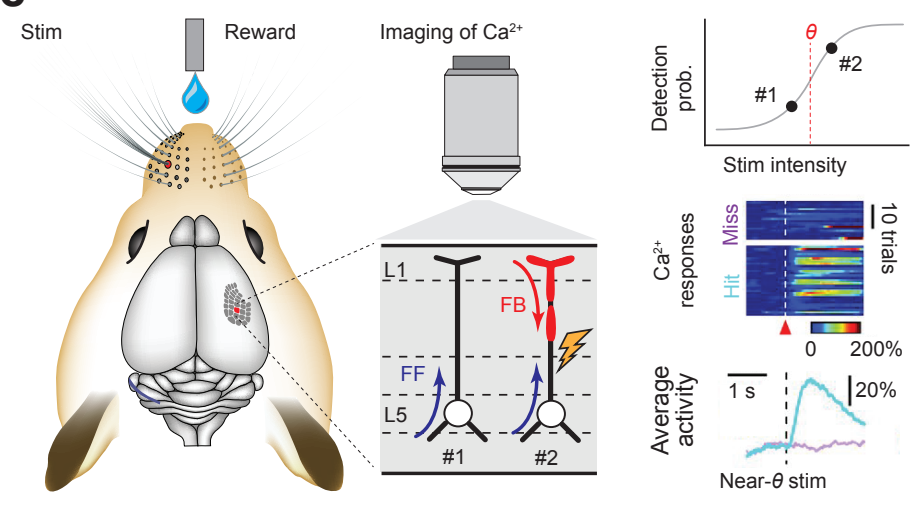


Fig. 6

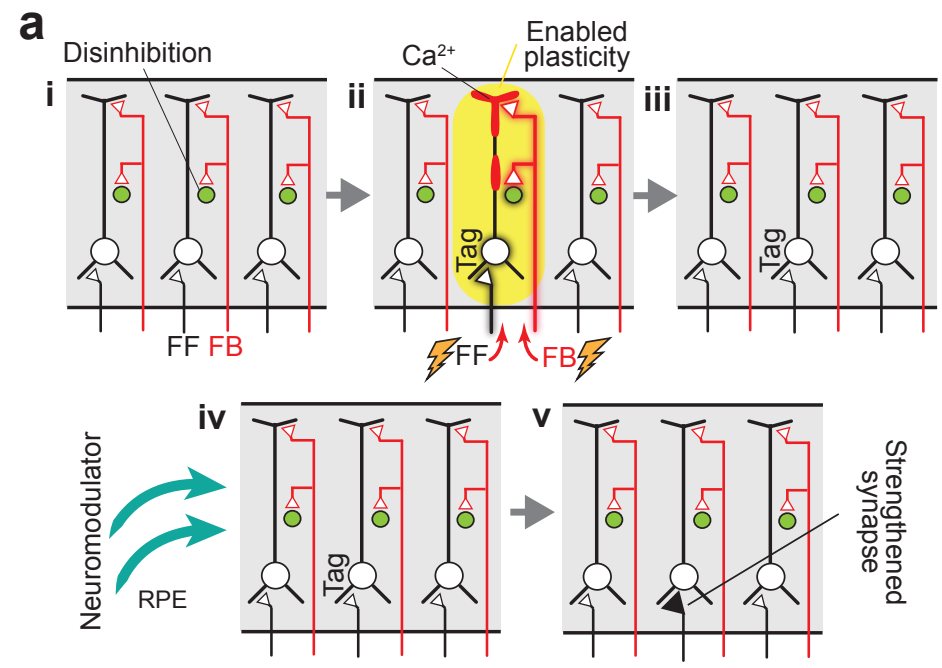

b

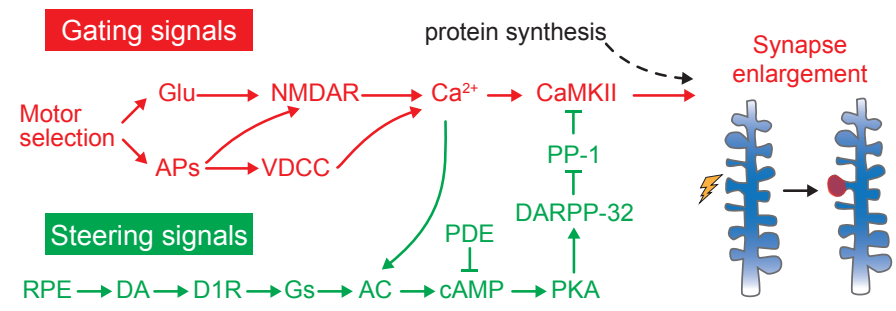




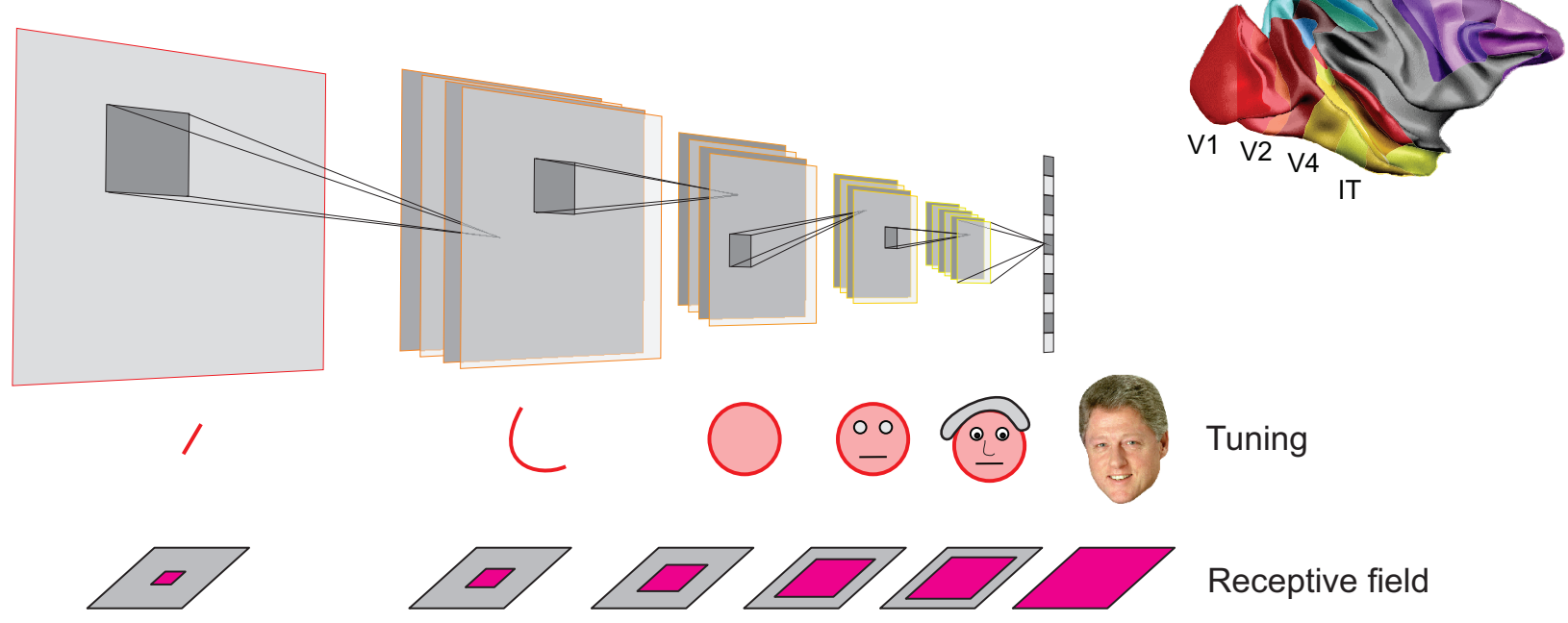

Fig. Box 